\title{
Origen y desarrollo del principio cooperativo de interés por la comunidad ${ }^{1}$
}

\author{
Daniel Hernández Cáceres²
}

Recibido: 25 de mayo de 2021 / Aceptado: 3 de junio de 2021 / Publicado: 29 de julio de 2021

Resumen. La Alianza Cooperativa Internacional en 1995, mediante la Declaración sobre la Identidad Cooperativa, realizó la última revisión mundial de los principios y valores cooperativos, reconociendo por primera vez como principio autónomo e independiente el principio de interés por la comunidad. Sin embargo, ante la práctica ausencia de estudios sobre el mismo, parece que dicho principio apenas ha alcanzado cierta relevancia para la comunidad científica. Esta falta de atención hacia el principio de interés por la comunidad puede generar ciertos problemas. En esa misma Declaración se establece que todos los principios están ligados entre sí, han de respetarse y han de estar presentes en las actuaciones de las cooperativas. Aplicando esta idea a la situación actual se puede deducir que, si no se estudia el principio de interés por la comunidad, es probable que no se aplique adecuadamente en las cooperativas, y si no se aplica, el resto de principios se resentirá.

A la vista de estas circunstancias, resulta de interés realizar un estudio del principio de interés por la comunidad que nos permita entender en qué consiste dicho principio y qué actuaciones forman parte del mismo. Para ello, se partirá del examen de su presunta presencia y aplicación en las experiencias e ideas de los precursores del cooperativismo moderno, como Owen, Fourier, King, Rochdale, Schultze-Delitzsch o Raiffeisen, entre otros, y se continuará con el análisis de su evolución en el seno de la ACI, hasta llegar a la interpretación actual del mismo.

Palabras clave: Desarrollo sostenible; Responsabilidad social; Identidad cooperativa; Precursores; Alianza Cooperativa Internacional.

Claves Econlit: M14; P13; Q01.

\section{[en] Origin and development of the cooperative principle of concern for community}

\begin{abstract}
In 1995, the International Co-operative Alliance, through the Statement on the Cooperative Identity, carried out the last worldwide revision of the cooperative principles and values, recognising for the first time the principle of concern for community as an autonomous and independent principle. However, given the virtual absence of studies on this principle, it seems that this principle has scarcely achieved any relevance in the scientific community.

This lack of attention to the principle of concern for community may lead to certain disadvantages. The same Statement states that all the principles are interlinked, must be respected and must be present in the actions of cooperatives. Applying this idea to the current situation, it can be deduced that if the principle of concern for community is not investigated, it will probably not be properly applied in cooperatives, and if it is not applied, the rest of the principles will suffer.

In view of these circumstances, it is of interest to carry out a study of the principle of concern for community in order to understand what this principle consists of and what actions form part of it. To this purpose, we will start by examining its presumed presence and application in the experiences and ideas of the precursors of modern cooperativism, such as Owen, Fourier, King, Rochdale, Schultze-Delitzsch and Raiffeisen, among others, and we will continue with an analysis of its evolution within the ICA, until we arrive at the current interpretation of the principle.
\end{abstract}

Keywords: Sustainable development; Social responsibility; Cooperative identity; Pioneers; International Cooperative Alliance.

Sumario. 1. Introducción. 2. El principio de interés por la comunidad en la génesis del cooperativismo. 3. La ACI y el principio de interés por la comunidad. 4. Consideraciones finales. 5. Referencias bibliográficas.

Cómo citar. Hernández Cáceres, D. (2021) Origen y desarrollo del principio cooperativo de interés por la comunidad. REVESCO. Revista de Estudios Cooperativos, vol. 139, e76634. https://dx.doi.org/10.5209/reve.76634.

1 Estudio realizado en el marco del Proyecto de I+D+i de generación de conocimiento «frontera» del Plan Andaluz de Investigación, Desarrollo e Innovación (PAIDI 2020) titulado "La reformulación de los principios cooperativos y su adaptación estatutaria para satisfacer las actuales demandas sociales, económicas y medioambientales" (P20_01278).

Este artículo forma parte de la Tesis Doctoral titulada: "El principio cooperativo de interés por la comunidad".

2 Universidad de Almería, España. Miembro del Centro de Investigación (CIDES) de la Universidad de Almería.

Contratado predoctoral perteneciente al programa de Formación del Profesorado Universitario del Ministerio de Ciencia, Innovación y Universidades (FPU18/04123)

Dirección de correo electrónico: dhernandez@ual.es. 


\section{Introducción}

Mediante la Declaración sobre la Identidad Cooperativa de 1995, la Alianza Cooperativa Internacional (ACI) realizó la última "revisión mundial de los principios y valores cooperativos sobre los que las cooperativas basan sus actividades, con el fin de fortalecer la identidad y el papel de las cooperativas en la economía global" (ACI, 1995:11), actuación que supuso la instauración de los actuales siete principios cooperativos, los cuales son: adhesión voluntaria y abierta; gestión democrática por parte de los socios; participación económica de los socios; autonomía e independencia; educación, formación e información; cooperación entre cooperativas; interés por la comunidad. Sobre ellos, esa misma Declaración establece que "los principios, que constituyen el centro de las cooperativas, no son independientes el uno del otro. Están unidos sutilmente; cuando se pasa uno por alto, todos se resienten" (ACI, 1995:45). Es decir, todos los principios han de respetarse y han de estar presentes en las actuaciones de las cooperativas. De manera que si una cooperativa no cumple con alguno de los principios, el resto de principios se resentirán, e incluso dependiendo de la trascendencia del incumplimiento puede dar lugar a que dicha entidad no encaje dentro de la llamada identidad cooperativa y que guarde más similitudes con otro tipo de forma jurídica.

Comprobar si una cooperativa respeta y aplica los principios cooperativos puede ser una tarea relativamente sencilla con respecto a algunos de ellos como el de puerta abierta, gestión democrática o participación económica. Estos principios están presentes casi desde la creación de la misma ACI, la cual se ha pronunciado acerca de su modificación y reinterpretación en múltiples ocasiones a lo largo de su historia, además de haber sido objeto de múltiples estudios científicos que han ido perfilando su interpretación. Sin embargo, no ocurre lo mismo con el principio de interés por la comunidad debido, en primer lugar, a su relativa "juventud", tratándose de un principio que, aunque la ACI advierte que al igual que el resto de principios está presente en el cooperativismo desde sus inicios, fue reconocido por primera vez como principio autónomo e independiente en esa Declaración de 1995. Y en segundo lugar, por la escasa relevancia que ha adquirido tanto para la ACI, la cual ya desde un momento inicial en el mismo informe que acompaña a la Declaración, únicamente le dedica un párrafo a ampliar y clarificar la interpretación de dicho principio, mientras que al resto de principios le dedica como mínimo una página completa; como para la comunidad científica, donde apenas existen estudios doctrinales sobre el mismo ${ }^{3}$. La ausencia de estudios doctrinales con respecto al interés por la comunidad, contrasta con la numerosa cantidad de literatura científica que se ha publicado en los últimos años sobre la responsabilidad social corporativa (RSC), la cual guarda una estrecha relación con este principio cooperativo. Esta falta de atención tanto por parte de la ACI, como de la comunidad científica, puede generar un grave problema, ya que si no se estudia este principio es probable no se aplique adecuadamente en las cooperativas, y si no se aplica, el resto de principios se resiente, provocando el problema de identidad anteriormente descrito.

A la vista de todas estas circunstancias, resulta de interés realizar un estudio doctrinal del principio de interés por la comunidad, partiendo del examen de su presunta presencia y aplicación en las primeras cooperativas, analizando la evolución de su interpretación e importancia en el seno de la ACI, hasta finalmente llegar a la interpretación actual del principio.

\section{El principio de interés por la comunidad en la génesis del cooperativismo}

A pesar de que la ACI admite que los principios cooperativos están presentes en el cooperativismo desde su inicio, el reconocimiento del interés por la comunidad no se realizó hasta el año 1995, esto es, 100 años después de la creación de la ACI y 150 desde la creación de la cooperativa de Rochdale, la cual, como veremos, es considerada por la doctrina como la primera cooperativa. Por ello, resulta de interés comprobar, en primer lugar, si realmente las primeras cooperativas aplicaban este principio en sus actuaciones y la forma en la que lo hacían.

\footnotetext{
Algunas de las más recientes publicaciones dedicadas al estudio de la identidad cooperativa y sus principios apenas le dedican unas páginas a este séptimo principio. Así, por ejemplo, en el libro de Macías Ruano, J.A. (2013) Las sociedades cooperativas y la adaptación de sus principios al mercado, aunque el epígrafe dedicado a este principio cuenta con 18 páginas, estrictamente hablando, al principio de interés por la comunidad únicamente le dedica 2 de las casi 250 páginas que tiene el libro. Esto mismo sucede en el libro de Martínez Charterina, A. (2016) La cooperativa y su identidad, el cual contiene una página y media destinada a la explicación de dicho principio de las 104 páginas que tiene el libro. Igualmente, en el número especial de CIRIEC-España. Revista jurídica de economía social y cooperativa, №27 de 2015 , dedicado a Principios y valores cooperativos en la legislación, de los 13 artículos que contiene, ninguno está dedicado a este séptimo principio.

De la misma forma, encontramos una total ausencia de estudios sobre esta materia si se realiza una búsqueda en algunas de las bases de datos científicas españolas más populares. Así, la búsqueda de los términos "interés por la comunidad” y otras combinaciones posibles con la palabra "cooperativa" y sus variantes (cooperativismo, cooperativista, etc.) en Dialnet no arroja ningún resultado que analice en exclusiva este principio. Lo mismo ocurre en el Catálogo de la Red de Bibliotecas Universitarias (Rebiun), no obteniendo ningún resultado relacionado con el principio cooperativo cuando se buscan los términos "interés por la comunidad".
} 


\subsection{Los precursores del movimiento cooperativo}

Habitualmente el inicio del cooperativismo se suele situar con la creación de Sociedad Equitativa de los Pioneros de Rochdale en 1844, pero es evidente que esta cooperativa no surge de manera espontánea. Existe un movimiento doctrinal previo conformado por varios autores, que con sus ideas y la puesta en práctica de las mismas a través de las primeras experiencias cooperativas del siglo XIX, con un mayor o menor éxito, tratan de configurar un tipo de sociedad que sirva para cubrir las necesidades que surgen en la clase obrera provocadas por el sistema capitalista imperante en la época ${ }^{4}$.

A continuación, se van a exponer las ideas y experiencias prácticas de esos precursores del cooperativismo ${ }^{5}$, tratando de identificar a su vez aquellas actuaciones que realizaban y que podrían encuadrarse como ejercicios del principio de interés por la comunidad, al no beneficiar exclusivamente a los miembros de sus organizaciones.

\subsubsection{Robert Owen}

Robert Owen (1771-1858) ${ }^{6}$ es considerado por muchos como el padre del cooperativismo en Inglaterra ya que establece algunos de los principios cooperativos que han llegado hasta nuestros días como son el de adhesión voluntaria y democrática, de retribución al capital mediante un interés limitado, de neutralidad política y religiosa y de promoción de la educación, y todo ello a través de empresas sin finalidad lucrativa, sino con la intención de servir a las necesidades de sus socios. Owen con sus organizaciones no buscaba exclusivamente una solución al problema de la distribución, sino que perseguía una solución total que abarcara también los problemas de la producción, de la educación y de la vida. Tenía un propósito de transformación social, de sustitución del sistema capitalista por otro que no estuviera basado en la competencia (Monzón Campos, 1989). Y es en la búsqueda de este sistema cuando emplea por primera vez el término cooperación, pero no en el sentido que le damos hoy en día, sino considerando la cooperación mutualista como un concepto totalmente opuesto a la competencia individual.

A lo largo de la vida de Robert Owen se identifican tres etapas. La primera coincide con sus actuaciones protectoras llevadas a cabo en su industria de New-Lanmark en Escocia, donde establece para sus obreros mejoras laborales, económicas y sociales, por ello esta etapa es considerada como una etapa "industrial paternalista" (Lambert, 1961:33). Sin embargo, no está convencido de que este paternalismo sea una solución suficiente para solventar el problema social, por ello, da un paso más allá y propone la creación de aldeas cooperativas especializadas en la agricultura o la industria, donde se organizara la producción y el consumo en común, y que estuvieran basadas en la igualdad y la propiedad colectiva. Así, en los años 20 establece la primera en Estados Unidos, New Harmony ${ }^{7}$, donde pone en práctica algunas de sus teorías como el principio democrático, la retribución del capital mediante intereses limitados, aunque con la intención de suprimirlos en el futuro, y la preocupación por la educación. Por último, la tercera de las etapas se corresponde con la constitución en 1835 de la Association of all Clases of all Nations, una organización creada para fomentar la creación de organizaciones comunitarias que lograran reformas pacíficas y que contribuyeran a poner en práctica un sistema de propiedad colectiva constituido por los miembros de la sociedad, sin lesionar los derechos de la propiedad existentes ${ }^{8}$.

Como hemos expuesto, Owen se interesó por la comunidad a través de la sustitución del sistema basado en la competencia por otro fundamentado en la cooperación que beneficiara a la comunidad en su totalidad.

Estas primeras cooperativas surgen a principios del siglo XIX, una época difícil para aquellas personas que pertenecían a la clase obrera. El proceso de revolución industrial ya se había trasladado desde Inglaterra al resto del continente europeo, consiguiendo la mejora de la producción de las fábricas a la vez que se generaba una sociedad dividida en clases sociales muy distanciadas económicamente. Por un lado, los empresarios, los cuales se dedicaron a acumular capital y cada vez eran más ricos; y por el otro, una clase obrera sobreexplotada, trabajando en jornadas de 16 horas durante los 7 días de la semana, con un salario reducido, sin ningún tipo de seguridad social que les permitiera cobrar un salario cuando enfermaran, sin indemnización por el habitual despido que se producía cuando alcanzaban cierta edad o cuando no eran rentables para la empresa, y donde era frecuente encontrar a niños de 12 años trabajando en minas y a mujeres embarazadas trabajando en jornadas de 12 horas en tareas agotadoras. A estas condiciones laborales, se han de sumar las condiciones en las que vivían fuera de la fábrica, con malos alojamientos, sin los servicios adecuados, con tiendas de barrio que ofrecen productos de dudosas calidades, medidas inexactas y precios elevados. En este contexto las clases obreras descubren que la actuación individual es insuficiente y que la única posibilidad de hacer frente a estas circunstancias es a través de la asociación. Por ello, para mejorar no solo las condiciones de vida, sino establecer un nuevo orden social y económico, los obreros deciden organizarse casi simultáneamente en tres ámbitos. En el ámbito laboral surge el sindicalismo como defensa ante las condiciones de trabajo impuestas; en el ámbito político surge el socialismo para influir en el poder, y finalmente, en materia socioeconómica nace el cooperativismo. Para una mayor profundidad sobre el contexto socioeconómico de las primeras cooperativas se puede revisar Martínez Charterina (2016).

5 En este trabajo me es imposible recoger las ideas de todos aquellos teóricos que influyeron con sus ideas en la aparición de las primeras cooperativas modernas, aun así, no podemos dejar de nombrar a teóricos como P.C. Plockboy y John Bellers, auténticos pioneros en el siglo XVII, y a otros muchos como Saint-Simon, William Thompson o J.H. Pestalozzi.

6 Para más información acerca la vida de Robert Owen y su contribución al mundo cooperativo puede consultarse la obra de Knopf, A.(1920) The life of Robert Owen by himself o el libro de Tsuzuki, C. (1992) Robert Owen and the World of Co-operation.

Esta aldea tiene corta duración, Owen confió demasiado en la ayuda del Estado y filántropos, lo que le llevó al fracaso (Gaminde Egía, 2015).

8 Esta organización ha sido considerada como el antecedente de la Alianza Cooperativa Internacional. Esta correlación entre ambas entidades es realizada por varios autores, entre otros Mladenatz (1969) y Lambert (1961). 
Para ello incluso llega a considerar que los excedentes de la cooperativa no deberían distribuirse entre los socios, sino que tendrían que acumularse en un fondo común que sirviera al desenvolvimiento de la sociedad o a la creación de otras cooperativas, contribuyendo de este modo a los fines generales del movimiento cooperativista y a la cooperación total ${ }^{9}$.

Además, Owen también realizó actuaciones a lo largo de su vida que son un reflejo de su preocupación por la sociedad. Así, entre estas actuaciones cabría destacar las realizadas en materia laboral en la primera de las etapas, donde redujo la jornada laboral de diez a siete horas, aumentó los salarios, comenzó a no contratar a niños de menos de diez años, creando escuelas gratuitas para ellos, y tuvo una gran influencia en la aprobación en 1819 de la primera Ley que reguló la contratación de menores. Igualmente, en esta misma fábrica decidió que "los beneficios pasarían a las reservas y se emplearían en interés de los trabajadores y del público" (Kaplan de Drimer \& Drimer 1975:209), construyó viviendas dignas para los operarios y creó comedores y lugares de aprovisionamiento donde abarató los artículos para el consumo. La implementación de estas actuaciones en su gestión empresarial procuró no solo una mejora de las condiciones de vida de los obreros, sino que también supuso un aumento de la producción y de los beneficios de la empresa.

\subsubsection{Charles Fourier}

Charles Fourier (1772-1837 $)^{10}$ fue el principal promotor del cooperativismo en Francia. Podría decirse que Francia tenía a Fourier e Inglaterra a Owen, los cuales, por cierto, mantuvieron varios contactos intercambiando proyectos e ideas a través de correspondencia ${ }^{11}$. A diferencia de Owen, él no busca una solución a los problemas del sistema, sino que simplemente trata de resolver un problema de producción y las imperfecciones de la distribución de mercancías (Lambert, 1961). Para ello, propuso que entre unas 1500 y 2000 personas se asociaran y compartieran vida y trabajo en una colonia en la que se mantendría la propiedad individual. A esta colonia la llama falansterio y sus miembros actuaban como trabajadores, capitalistas y consumidores, por lo que albergaban intereses contrapuestos. Fourier trató de que a través de la ayuda mutua se alcanzara la armonía de intereses de las diferentes clases sociales, de forma que se conformara una red completa de trabajo y consumo comunitario basada en las solidaridades múltiples (Monzón Campos, 1989). Es en estos falansterios donde desarrolla algunos de los principios actuales, como son el de adhesión voluntaria, funcionamiento democrático ${ }^{12}$ y el retorno cooperativo, del cual podría ser considerado el precursor, ya que suprime los salarios, recibiendo los trabajadores por su trabajo únicamente un dividendo en función del trabajo realizado. Sin embargo, a diferencia de Owen, remunera el capital de forma proporcional al resultado de la actividad del falansterio ${ }^{13}$.

El interés por la comunidad en Fourier se muestra con claridad en su preocupación por las condiciones laborales de los trabajadores de sus falansterios, atribuyéndosele el título de ser la primera persona en señalar el derecho de todos los ciudadanos al trabajo (Mateo Blanco \& Palacio, 1979), debiendo pasar este de ser considerado como una necesidad o una condena, a convertirse en un placer. Este autor partió de la idea de que el trabajo cuanto más placentero sería más productivo ${ }^{14}$. Para ello mejoró la distribución y las condiciones higiénicas de los talleres y permitió que los trabajadores se organizaran según sus afinidades pudiendo elegir los trabajadores sus labores preferidas e incluso alternar entre diferentes tareas ${ }^{15}$.

Además de este interés por las condiciones laborales, Fourier se preocupa de otros aspectos que afectan a la comunidad dentro de sus falansterios, como es la mejora de la higiene, la distribución urbana y la dotación de servicios, con comedores, viviendas, salas de lectura, de juegos, de teatro, etc. e incluso propone la construcción de viviendas gratuitas para las clases menos pudientes.

9 Tal y como señalan varios autores, con esta concepción, se desalentaba la adhesión de miembros a la cooperativa, ya que no obtenían desde un punto de vista empresarial ningún beneficio directo de la participación en la misma, y su integración obedecía más a aspectos morales (Monzón Campos, 1989; Divar Garteiz-Aurrecoa, 2012).

10 Para ampliar la información acerca de las ideas de Charles Fourier puede consultarse su obra Fourier, C. (1966) Théorie de l'unité universelle.

11 Sobre el contacto de Fourier y Owen puede verse a Benevides Pinho (1987).

12 En el falansterio el cargo de dirección sería elegido entre todos los miembros, debiendo someterse anualmente las personas que ostentaran estos cargos a una crítica o debate con veinte miembros que tuvieran antipatía con el candidato, en la que se evaluaría la gestión del mismo. Por lo que el cargo del dirigente correría peligro en caso de que dejara de tomar en consideración los intereses de todos los miembros del falansterio.

13 Este hecho hizo que Lambert le tildara de "presocialista" (Lambert, 1961:38).

14 En palabras de Fourier: "Para que el trabajo resulte atrayente, la industria societaria deberá reunir las siete condiciones siguientes: 1) Que cada trabajador esté asociado y que su retribución sea por dividendo y no por salario; 2) Que cada uno, hombre, mujer o niño, sea retribuido con relación a sus condiciones: capital, trabajo y talento; 3) Que los períodos de trabajo industrial sean cambiados alrededor de ocho veces por día: el interés en el desempeño de una función agrícola o manufacturera no puede mantenerse más de una hora y media o dos horas; 4 ) Que ellas sean ejercidas con la compañía de amigos espontáneamente reunidos, y despertando su curiosidad y estimulados por una emulación muy activa; 5) Que los talleres y lugares de cultivos ofrezcan al obrero el incentivo de la elegancia y del aseo; 6) Que la división del trabajo sea elevada a tal grado para que resulte del agrado de cada sexo y edad y acorde con las funciones para las cuales sea conveniente; 7) Que dentro de esta distribución, cada uno, hombre, Mujer o niño, goce plenamente del derecho al trabajo y del derecho a intervenir cuando lo desee en la clase de trabajo que quiera elegir, con la sola justificación de su probidad y aptitud" (Mladenatz, 1969:43).

15 Kaplan de Drimer \& Drimer (1975:212) manifiestan que Fourier "es uno de los primeros expositores o partidarios de la economía planificada, del trabajo agradable, de la consideración hacia las inclinaciones naturales de los trabajadores y de la seccionalización de las tareas”. 


\subsubsection{William King}

William King (1786-1865) destaca, además de por su clara intención de reforma social a través del asociacionismo obrero, por promover el carácter voluntario y la neutralidad religiosa del cooperativismo ${ }^{16}$. Consciente de las circunstancias de miseria en las que viven las clases obreras y de la dependencia que estos tienen del capital ajeno, propone su liberación a través de la auto ayuda (self help) entre trabajadores. Para ello, plantea que inicialmente constituyan una cooperativa de consumo, consiguiendo a través de ella ahorrar de forma colectiva las ganancias que normalmente obtienen los comerciantes e intermediarios cuando compran en sus establecimientos de consumo. Este ahorro les permitiría acumular colectivamente el capital y posteriormente invertirlo en la creación de empresas de producción propias, donde trabajarían estos obreros en su propio beneficio ${ }^{17}$. De esta manera se observa como King no solo concibe las cooperativas como un medio para aumentar la renta de los trabajadores, sino como una herramienta de transformación de la sociedad. King llevó sus teorías a la práctica al crear en 1827 la cooperativa de consumo The Cooperative Trading Association en Brighton, con la que obtuvo bastante éxito, reproduciéndose rápidamente por todo el país, apareciendo más de trescientas nuevas cooperativas, llegando incluso a celebrar estas varios congresos entre los años 1831 y 1835. Además, dentro de sus actuaciones en pro del cooperativismo, cabría destacar la redacción en solitario del periódico mensual The Co-operator durante los años 1828 y 1830.

El interés por la comunidad en King se identifica en su teoría de transformación social a través del cooperativismo. Se preocupa por los obreros y busca, inicialmente mediante el asociacionismo de consumo y posteriormente mediante el de producción, la transferencia de los medios de producción en manos de la propiedad privada, a las manos de la propiedad cooperativa. Para alcanzar este fin, al igual que Owen, destina los excedentes obtenidos a la producción propia y a la adquisición de propiedades agrarias, contribuyendo a su vez a alcanzar el ideal de transformación social. De forma que puede decirse que sus cooperativas trabajaban para la sociedad en general, tratando de sustituir el régimen capitalista basado en el lucro por otro de cooperación.

\subsubsection{Jean-Philippe Buchez}

Jean-Philippe Buchez (1796-1865) es otro de los principales teóricos franceses de principio de siglo que establecieron las bases de las posteriores experiencias cooperativas de producción. En su célebre artículo publicado en el Journal des sciences morales et politiques del 17 de diciembre de 1831 titulado Medio de mejorar la condición de los asalariados de las ciudades", expone su proyecto para "mejorar la condición de los obreros libres y para introducir la propiedad y la buena fe en los mercados" (Lambert, 1961:321). En dicho artículo establece un programa a seguir por los obreros en el que se deducen algunos de los principios cooperativos actuales como el de democracia, puerta abierta y retorno cooperativo en proporción a su trabajo. Buchez puso en práctica estas ideas con la creación en 1832 de una cooperativa de producción formada por ebanistas que tuvo que ser liquidada al poco tiempo, y en 1834 con la constitución de $L a$ asociación de los joyeros en dorado la cual pervivió hasta 1873.

En varias de las normas de las organizaciones proyectadas por Buchez y expuestas en su célebre artículo, se deduce la gran preocupación por la comunidad que han de mostrar este tipo de entidades y que es interiorizada en su funcionamiento. Así, el tercer principio de sus normas indica que una parte de los beneficios podría ser empleado "el mantenimiento de ancianos, enfermos e impedidos, así como para aliviar la crisis de organizaciones industriales semejantes"19 (Gaminde Egia, 2015:58). Además, en caso de disolución de la entidad, el capital social acumulado en las reservas durante sus años de vida, debería destinarse a las reservas de otra sociedad cooperativa, a una obra filantrópica, o al Estado. Finalmente, también se preocupa de eliminar una posible explotación laboral sobre los trabajadores que no son parte de la asociación al establecer la prohibición de contratar a obreros no socios durante más de un año ${ }^{20}$.

16 El principio de la neutralidad se encuentra mejor recogido en las cooperativas de King que en la Cooperativa de Rochdale. En este sentido, la resolución del congreso celebrado por las cooperativas que siguieron este modelo en 1832 decía: "como el mundo cooperativo agrupa personas de todas las sectas religiosas y de todos los partidos políticos, se acuerda por unanimidad que los cooperadores, en cuanto tales, no se encuentran identificados con ningún dogma religioso, irreligioso o político" (Lambert, 1961:39).

17 El 1 de agosto de 1828 escribió en The Co-operator: "Las clases obreras tienen un motivo de mucha importancia para abrir tiendas para ellas mismas. La cantidad de dinero que las clases obreras gastan anualmente es enorme. Puede cifrarse en varios millones. El beneficio realizado sobre esta suma sería suficiente, por sí mismo, para establecer no pocas fábricas. No es la falta de poder, sino la falta de saber, lo que impide a los trabajadores ponerse manos a la obra y empezar... Comerciar para ellos mismos y trabajar para ellos mismos les produciría beneficios, como consecuencia, capital, y, a su vez, como consecuencia de éste, la independencia" (Lambert, 1961:40).

18 El artículo al completo se puede encontrar en la publicación de Lambert (1961)

19 Concretamente establece que "una cantidad equivalente a la que los empresarios intermediarios descuentan cada jornada, se reservaría; a fin de año, esa cantidad, que sería el beneficio neto, se repartiría de la siguiente forma: veinte por ciento para formar y aumentar el capital social; el resto se emplearía en socorros o se distribuiría entre los asociados, a prorrata de su trabajo" (Lambert, 1961:321).

20 El quinto de sus principios indica que "La asociación no podría hacer trabajar por su cuenta a obreros extraños durante más de un año; pasado este tiempo, estaría obligada a admitir en su seno al número de trabajadores nuevos que se hubieran hecho necesarios por el aumento de sus operaciones" (Lambert, 1961:321). 


\subsubsection{Michel Derrion}

Michel Derrion (1802-1850) es quizás uno de los pioneros del cooperativismo más desconocidos. Seguidor de las ideas de algunos de sus predecesores como Fourier, King o Saint-Simon, centra sus teorías y prácticas en la reforma y el cambio social a través del asociacionismo de consumo de los trabajadores, el cual permitiría en el futuro pasar a la organización de la industria ${ }^{21}$. Estas ideas las expone inicialmente a través del periódico que adquiere, L'Indicateur, y posteriormente, en 1835, con la creación de la tienda cooperativa llamada Commerce veridique et social: Derrion et compagnie, creada con suscripciones voluntarias, incluida gran parte de su fortuna ${ }^{22}$. En esta tienda pone en práctica algunos de los principios cooperativos como el de distribución de excedentes en proporción a sus operaciones, el de puertas abiertas y el de la participación de los asociados consumidores en la gestión de la misma. Sin embargo, al igual que Fourier, establece la retribución al capital mediante unos porcentaje de los beneficios de la empresa.

Derrion en sus teorías tiene presente a la comunidad en su conjunto. Así, en sus artículos publicados en su periódico entre los años 1834 y 1835 propone la realización de un comercio verídico que contemple la previsión social y que dote un fondo social que permita realizar obras a favor de los menos favorecidos. Asimismo, en su empresa hace partícipes en la distribución de los beneficios a los trabajadores, otorgándoles una cuarta parte de los beneficios, y destinando otra cuarta parte a obras de bien común.

\subsubsection{Louis Blanc}

Por último, señalar también a Louis Blanc (1812-1882) ${ }^{23}$, periodista nacido en Madrid que popularizó en Francia el asociacionismo de producción entre las clases obreras gracias a sus famosos Talleres Sociales dedicados a la gran industria y que, tal y como ocurre con otros autores precedentes, también cree que es necesaria una transformación social. Blanc considera que todos los problemas sociales provienen del régimen de competencia vigente, por ello expone que "la solución al problema social en la actual situación económica debe buscarse en la organización del trabajo por medio de la asociación" tal y como recoge en su obra Organisation du Travail publicada en 1840 (Merino Hernández, 2005:185). Para desarrollar esta organización del trabajo deposita toda su confianza en la creación de los talleres sociales, unas asociaciones de obreros sobre los que se asentaría el nuevo sistema económico ${ }^{24}$. Dadas las dificultades de los obreros para acumular el capital, Blanc pensó que en un momento inicial fuese el Estado el que prestase el capital a los obreros para la constitución de estos talleres, siendo también el Estado el encargado de redactar los estatutos y designar a una persona para que actuase como dirigente. Sin embargo, a partir del primer año, los obreros comenzarían a tomar el control del taller, eligiendo ellos mismos a sus dirigentes y convirtiéndose en una cooperativa de trabajo autónoma.

Entre las ideas de Blanc, además de la sustitución del sistema competitivo por otro basado en la cooperación, se observa una clara preocupación por la comunidad. Así entre sus actuaciones cabría destacar la propuesta de que los beneficios de los talleres sociales, una vez reembolsados los préstamos estatales, se dividan en tres partes, la primera de las tres "se distribuiría entre todos los trabajadores y se sumaría así a sus salarios", y la segunda de ellas iría destinada a "socorrer ancianos, enfermos o inválidos y a aliviar las crisis que pudieran afectar a otras industrias" (Kaplan de Drimer \& Drimer, 1975:221).

\subsection{Experiencias prácticas cooperativas en el origen del cooperativismo}

Cabe señalar la dificultad de realizar una distinción entre precursores y primeras realizaciones del cooperativismo moderno, ya que no hay una fecha o un hecho determinante que permita distinguir entre ambas categorías. Algunas de las experiencias citadas en el apartado anterior pueden considerarse a su vez precursoras y primeras realizaciones, como por ejemplo las llevadas a cabo por Buchez, Derrion o King. Por ello, en este apartado nos centraremos en aquellas entidades e ideas que surgieron a partir de 1844, año en el cual se crea la que es considerada como la primera cooperativa, la cooperativa de Rochdale.

Tal y como expresa la ACI (1995:25) "no hay una raíz única de la que emana todo tipo de cooperativas", sino que fueron el conjunto de doctrinas y experiencias que se han visto en el epígrafe precedente las que

21 En 1835 escribe en su periódico: “TTrabajadores!... Sois productores. Pero ¿no sois más que eso?. ..¡No sois también consumidores? ¡Entonces!... En esta cualidad de consumidores reside el poder de que os hablaba hace un momento. No busquéis en otras partes medios de acción, pues es ahí donde se encuentra vuestra única fuerza reformadora. Como consumidores, poseéis la palanca que Arquímedes pedía para mover el mundo. Sólo hace falta que aprendáis a serviros de ella.” (Lambert, 1961:41)

22 Tras los ataques recibidos por los comerciantes, las autoridades declararon sus finalidades ilícitas y ordenaron su clausura, durando solo tres años.

23 Para ampliar la información sobre este autor se pueden consultar otras obras como Loubère, Leo A. (1961) Louis Blanc his life and his contribution to the rise of French Jacobin-Socialism, o Humilière, J.M. (1982) Louis Blanc: 1811-1882.

24 Su idea era que, en una primera fase, estos talleres competirían contra las empresas privadas, siendo absorbidas estas últimas progresivamente por los talleres sociales; en una segunda fase todos los talleres sociales de una misma rama de la industria se agruparían alrededor de un taller central; y en una tercera fase, se produciría la solidaridad de todas las industrias. 
configuraron y desarrollaron algunos de los actuales principios cooperativos como el de asociación, de voluntariedad, de democracia, de actividad para el servicio, de retorno a prorrata de las compras y, muy especialmente, el de interés por la comunidad, al presentar una gran vocación social y preocupación por la comunidad en la que se insertaban. Fueron las ideas de ese conjunto de autores las que sentaron las bases para que pudiera desarrollarse, a partir de mediados del siglo XIX, las que hoy en día se conocen como las primeras experiencias cooperativas. La más famosa de todas ellas es la cooperativa de consumo de Rochdale, pero a su vez, en el resto del continente europeo surgieron múltiples cooperativas que abarcaron otras ramas de la actividad económica y satisficieron necesidades muy dispares como el crédito, el trabajo, la colocación de la producción, el aprovisionamiento de artículos o servicios, etc.

La clasificación de estas primeras experiencias ha sido realizada de manera muy dispar entre los distintos autores ${ }^{25}$, pero en todas esas clasificaciones encontramos ciertos nombres y experiencias cooperativas que se repiten, como Rochdale, Schulze-Delitzsch, Raiffeisen, Haas, Luzzatti, Desjardins, Fourier, Buchez y Blanc. En nuestro caso, clasificaremos estas experiencias en cuatro tipos diferenciados de cooperativas: cooperativas de trabajo ${ }^{26}$, cooperativas de colocación de la producción y de provisión de artículos o servicios, cooperativas de consumo y cooperativas de crédito. En base a dicha clasificación, en este apartado vamos a tratar de analizar si estas cooperativas continuaron con las prácticas de preocupación por la comunidad presentes en sus predecesores, o si, por el contrario, se centraron en satisfacer únicamente las necesidades de sus socios y en lograr objetivos exclusivamente económicos.

\subsubsection{Cooperativas de colocación de la producción y de provisión de artículos o servicios}

Dentro de esta rama del cooperativismo se situarían, por ejemplo, las actuales cooperativas agroalimentarias, que fueron creadas para satisfacer las necesidades de los agricultores y ganaderos, o las cooperativas de servicios, que agrupaban a pequeños artesanos y comerciantes.

Entre los principales precursores de las cooperativas agrícolas podríamos situar a Wilhelm Haas (18391931), pionero que también tuvo un papel importante dentro del cooperativismo de crédito. Haas inició su primera cooperativa en 1872 en la región alemana de Hesse, a través de la cual trató de mejorar las condiciones económicas de los agricultores mediante la provisión en común, suministrando artículos para las tareas agrícolas a un precio inferior. Tras estas primeras experiencias, trasladó su ideario cooperativo a las centrales lecheras y a las cajas de crédito rural. Este ideario fue recogido en 1890 en el llamado Programa de Darmastadt, proponiendo medidas intermedias entre las propuestas por Raiffeisen y Schultze-Delitzsch ${ }^{27}$.

En Dinamarca también existió un fuerte desarrollo de este tipo de cooperativas, inspiradas en las ideas de Nikolai F. S. Grundtvig. Creadas por los agricultores para principalmente facilitar la exportación de sus productos, también realizaron actividades de provisión y venta en común, así como de uso en común de maquinarias. La organización de las cooperativas se realizaba por especialidades, siendo las cooperativas lácteas la más antiguas y las que gozaron de un mayor desarrollo. Entre las normas de funcionamiento de dichas cooperativas se encuentra la obligación de los socios de entregar toda su producción excepto la destinada al consumo familiar, la fijación del capital que cada socio debía aportar se realizaba en proporción a la cantidad de ganado que poseían, el beneficio se repartía a razón de la cantidad de leche suministrada y los socios podían abandonar la cooperativa cumpliendo un plazo de preaviso de un año.

En Francia este tipo de cooperativas se desarrollaron con una estrecha vinculación a los sindicatos agrícolas. Estos sindicatos se creaban sin un capital social, por lo que en ocasiones, a modo de secciones

25 Unos han establecido diferentes modelos cooperativos, otros hablan de sistemas cooperativos, mientras que otros simplemente han clasificado estas experiencias en función de la actividad económica que desarrollan. La ACI (1995) las clasifica en cooperativas de consumo, de trabajo, de crédito, agrícolas y de servicios; Mladenatz (1969) habla de la existencia de tres sistemas cooperativos fundamentales que quedan vinculados a tres nombres: Rochdale, Schulze-Delitzsch, y Raiffeisen, los cuales conviven con otros sistemas intermedios, los de Haas, Luzzatti, el sistema danés, las cooperativas agrícolas en Francia y el Boerenbond belga; por otro lado, Lambert (1961) distingue entre la cooperación conectada con un plan de reorganización de la sociedad, donde introduce a Rochdale, la cooperación considerada con independencia de un plan de reorganización, en la que incluye a Schulze-Delitzsch y Raiffeisen, y el socialismo del consumidor, en el que incluye a pensadores posteriores a Rochdale y a todas esas primeras experiencias cooperativas; por su lado Benevides Pinho (1987:45) señala "tres modelos cooperativistas a partir de los cuales son elaboradas todas las variaciones posibles de cooperativas", estos son: el modelo de consumo, donde incluye a Rochdale, el modelo cooperativista de producción, en el que nombra a Buchez, a Fourier y a Blanc, y los modelos cooperativistas de crédito en los que incluye a Schulze-Delitzsch, a Raiffeisen, a Luzzatti y a Haas; y finalmente, Kaplan de Drimer \& Drimer (1975) hablan de cooperativas de consumo, en la que incluyen a Rochdale, cooperativas de crédito, donde nombran a Schulze-Delitzsch, Raiffeisen, Haas, Luzzatti, Wollemborg, cooperativas de trabajo, en la que mencionan a Buchez y Blanc, y cooperativas de colocación de la producción, y de provisión de artículos o servicios.

26 Hay que señalar que en este epígrafe no haremos mención a las cooperativas de trabajo ya que su desarrollo principal fue llevado a cabo en Francia por Buchez, Fourier y Blanc, autores a los que ya se ha hecho referencia en el apartado anterior, por lo que nos remitimos al mismo para conocer sus características y la presencia en su seno del interés por la comunidad.

27 Las notas fundamentales de dicho programa son recogidas por Kaplan de Drimer \& Drimer (1975:252) de manera resumida como: “cooperación basada en el esfuerzo propio y la ayuda mutua de los agricultores asociados, sin excluir la eventual ayuda del Estado; realización cooperativa de actividades diversas (provisión, colocación de la producción, crédito, seguros y otros servicios), pero a través de entidades cooperativas especializadas; radio de acción limitado para las cooperativas de primer grado; responsabilidad preferentemente ilimitada de los asociados en las cooperativas de crédito locales (o de primer grado); integración regional de las mismas, a través de federaciones (que realizan diversas funciones comunes de carácter económico) y de uniones (que desarrollan actividades de tipo técnico- social); y agrupación de las organizaciones regionales en una unión de carácter nacional". 
especializadas, constituían cooperativas agrarias que tenían la finalidad de llevar a cabo la transformación y venta de los productos, el aprovisionamiento de los elementos necesarios para las explotaciones agrícolas o la prestación de otros servicios comunes.

Las primeras cooperativas agrícolas belgas surgen de forma parecida a las francesas. En Bélgica, los agricultores estaban asociados a través de las guildas $^{28}$ agrícolas, la cuales también recurrían a la constitución de secciones para llevar a cabo actividades económicas, entre las que se encontraba la dedicada al suministro de artículos para sus miembros y a la venta en común de los productos de los agricultores. Las características y normas de funcionamiento de estas secciones muestran que se trataban de unas cooperativas tempranas.

Finalmente, con respecto a este cooperativismo de colocación de la producción y de provisión de artículos o servicios, también cabría destacar el papel protagonista que adoptaron algunos de los autores que veremos en el epígrafe destinado a las cooperativas de crédito como Schultze-Delitzsch, Raiffeisen o Luzzatti ${ }^{29}$.

A pesar de haber recogido algunas de las primeras experiencias de esta tipología cooperativa, en el desarrollo de esta investigación no se ha podido encontrar suficiente información como para extraer alguna conducta que muestre la preocupación por la comunidad en estas cooperativas, más allá de la búsqueda de la satisfacción de intereses a través de la mutualidad. Sin embargo, se ha decidido incluir este breve apartado dado que, en mi opinión, cobraba interés recoger aunque sea mínimamente el desarrollo de algunas de las primeras experiencias prácticas de esta tipología cooperativa, al igual que se va a realizar a continuación con las cooperativas de consumo y de crédito.

\subsubsection{Cooperativas de consumo: La sociedad de los Probos Pioneros de Rochdale}

Aunque no se trata de la primera cooperativa de consumo ${ }^{30}$, sin ninguna duda ha sido la más relevante de todas, sirviendo de ejemplo y estímulo a otros grupos sociales a la hora de asociarse a través de la forma de cooperativa. "El gran mérito de los cooperadores de Rochdale es el de haber codificado en cierta forma, en los estatutos que ellos se dieron, las mejores enseñanzas de la experiencia acumulada por los cooperadores que les precedieron y el de haber conducido sus negocios con un gran sentido práctico. Su éxito ha hecho de su sociedad el modelo seguido en todos los países para las cooperativas de consumo" (Fauquet, 1973:56). Entre sus aportaciones al movimiento cooperativo se encuentra el sistematizar un conjunto de normas y principios para el funcionamiento y organización de las cooperativas, la elaboración de un programa de acción cooperativo y el ser un ejemplo de iniciativa popular de carácter idealista y práctico que logró alcanzar un éxito extraordinario.

La cooperativa surge en $1844^{31}$ tras el intento infructuoso de un grupo de tejedores por conseguir un aumento de salario. Ante esta situación propusieron varias alternativas, como luchar por la Carta del pueblo, o el ahorro a través de abstenerse de consumir bebidas alcohólicas. En cambio, la opción que adoptaron fue la de asociarse a través de la acción común y emplear los medios a su alcance adoptando medidas tendentes al beneficio pecuniario y al mejoramiento de las condiciones sociales y domésticas de sus miembros, tal y como recoge el artículo 1 de los estatutos de $1844^{32}$.

Sin embargo, las actividades que se propuso desarrollar la cooperativa no tenían como objetivo final el beneficio exclusivo de sus miembros, sino que al igual que $\mathrm{Owen}^{33}$, el proyecto de Rochdale fue un proyecto de comunidad integral, en el que a través de la constitución de cooperativas se pretendía abarcar la totalidad de las actividades económicas. Todas estas actividades las realizarían en base a los principios de libre adhesión y salida de la cooperativa, neutralidad política y religiosa, gestión democrática por los socios, intereses limitados al capital social, retorno de los excedentes en proporción a las compras, compraventa al contado, pureza y calidad de los productos y la promoción de la educación.

Fueron 28 los Pioneros de Rochdale, debiendo señalar que entre todos ellos había una mujer, Ana Tweedale, algo que no era nada habitual en las sociedades de la época. Estos miembros se comprometieron a aportar 1 libra cada uno y, una vez que consiguieron acumular las 28 libras, alquilaron un bajo de una casa situado en Toad Lane (Callejuela del Sapo) donde comenzaron a desarrollar la actividad cooperativa. Tras

28 Las guildas era una de las formas de asociación a las que recurrían los grupos de artesanos y mercaderes durante la Baja Edad Media y que tenían como finalidad la ayuda mutua y la protección de los intereses de sus asociados.

29 Estos también trataron de facilitar el suministro de artículos y servicios que necesitaban los agricultores, pequeños artesanos y comerciantes, a la vez que se encargaron de promover la venta en común a través del sistema cooperativo planteado por cada uno de ellos. Aquellas cooperativas que se dedicaban a estas actividades y que se constituían acogiéndose a las normas establecidas por alguno de ellos, presentarán las mismas actuaciones de interés por la comunidad que las recogidas en los respectivos apartados dedicados a las entidades de crédito, por lo que nos remitimos a esos apartados para comprobar dichas actuaciones.

30 Mladenatz (1969) habla de que ya existían cooperativas de consumo en Inglaterra y en Francia, donde el movimiento cooperativo comenzó en Lyon, entre 1830 y 1850. Por su parte, Divar Garteiz-Aurrecoa (2011) señala que los Pioneros no eran tan pioneros, ya que cerca de Rochdale, en el condado de Lancaster ya había funcionado una cooperativa de consumo entre 1832 y 1835, incluso fecha la creación de la primera cooperativa de consumo en 1827 en la ciudad de Brigthon siguiendo la doctrina de William King.

31 La historia de la cooperativa se encuentra relatada detalladamente en la obra Historia de los pioneros de Rochdale por G.J. Holyoake (1973).

32 Los estatutos de Rochdale de 1844 y sus posteriores modificaciones se encuentran recogidos en la obra de Lambert (1961).

33 Entre los miembros seis eran discípulos de Owen (Monzón Campos, 1989), por lo que resulta evidente la influencia que ejercieron las ideas de este sobre la configuración de la cooperativa. 
esta tienda llegó la apertura de la sala de lectura, la biblioteca social, la constitución de la Sociedad del Molino Harinero en 1850, la Sociedad cooperativa Manufacturera de Rochdale en 1854 y la cooperativa de segundo grado Sociedad Cooperativa del Norte de Inglaterra para la Venta al por Mayor en 1863.

En los estatutos de la cooperativa de Rochdale, aunque no se establece expresamente, se percibe la preocupación por la sociedad, al hacer coincidir sus actuaciones con los intereses de la comunidad ${ }^{34}$. Así, la entidad incorpora en el artículo 1 de los estatutos la inquietud que tenía una parte de los primeros pioneros, los denominados teetotalers (abstemios), por el consumo de alcohol de los obreros, planteando inicialmente que el dinero que gastaban en estas bebidas podía destinarse a mejorar el bienestar de su familia. El interés por promover la sobriedad es adoptado por la sociedad, incluyendo entre las actividades a realizar por la cooperativa la apertura de "una sala de templanza tan pronto resulte conveniente, en una de las casas de la Sociedad" (Lambert, 1961:327). Asimismo, este artículo 1 también incluye el propósito de la transformación del sistema económico por un sistema basado en el cooperativismo que beneficiara a la comunidad en su totalidad $^{35}$. Por último, el artículo 44, tras la reforma estatutaria llevada a cabo en 1854, establece que en caso de disolución de la cooperativa el activo, una vez pagadas las deudas y devueltas las aportaciones a los socios, "se utilizará por los administradores de aquel momento en los fines caritativos o públicos que crean convenientes" (Lambert, 1961:333), lo que es una muestra más del evidente interés social de esta entidad.

Posteriormente, conforme la actividad de la cooperativa evoluciona, estas actividades en favor de la comunidad se van viendo ampliadas. Así, cuando en 1855 se crea la Sociedad Cooperativa Manufacturera de Rochdale, se propone la participación de los obreros en los beneficios, sin embargo, en palabras de John Brierley, secretario de la cooperativa en 1867, "los capitalistas socios pensaron que los trabajadores iban a recibir demasiados beneficios, y entonces resolvieron suprimir la parte reservada al trabajo" (Holyoake, 1973:107), por lo que la sociedad continuó trabajando aunque sin dicha participación en resultados. Además, durante la crisis de la guerra de secesión americana entre 1861 y 1864, parte de los beneficios de la cooperativa se destinaron a tareas de socorro y ayuda social, y en 1870, cuando la cooperativa contó con su propia sala para asambleas, organizó una serie de conferencias a las que pudieron acudir personas que no eran socias de la cooperativa, siendo la primera entidad de la ciudad en organizar actos de esta naturaleza.

Finalmente destacar la gran cantidad de actividades de carácter social que realizaron, como la creación de la Sociedad de Previsión para casos de Enfermedades y de Socorros para los Sepelios, que tenía como objetivo el suministrar subsidios a los enfermos y sufragar los funerales; o la apertura de la biblioteca y sus sucesivas ampliaciones, y la creación de escuelas, todas ellas costeadas con los fondos creados expresamente para la educación. Sin embargo, estas actividades fueron diseñadas para el uso exclusivo de sus socios, por lo que no se puede decir que en dichas actuaciones hubiera un interés especial por la comunidad, sino más bien un interés en el bienestar y la formación de los propios socios.

\subsubsection{Cooperativas de crédito}

\subsubsection{Hermann Schultze-Delitzsch}

Hermann Schultze-Delitzsch (1808-1883) es considerado el iniciador del cooperativismo en Alemania hasta el punto de que el sistema cooperativo que crea, y que en la actualidad sigue siendo una referencia, recibe el nombre de modelo Schultze-Delitzsch. Para este autor, los fines de la cooperación no están ligados a una reforma general del sistema capitalista ${ }^{36}$, sino que sus fines son exclusivamente económicos, tratando de mejorar las condiciones económicas de las clases medias urbanas, aumentando sus rentas y salarios a través de la asociación y sin ningún tipo de ayuda externa ${ }^{37}$. Así que a partir de 1849 comienza a crear asociaciones de pequeños comerciantes, artesanos e industriales tanto para la provisión de materias primas y la venta en común, como para el crédito, siendo estas últimas asociaciones las que alcanzaron un mayor éxito. En estas cooperativas se observan algunas de las características que mantienen las cooperativas actuales, como la ayuda mutua, la libre asociación, la conformación del capital exclusivamente por las aportaciones de los socios y el carácter democrático en la administración de la cooperativa, la cual es llevada por los socios. Sin embargo, presenta importantes diferencias con el cooperativismo actual, como la distribución de beneficios

34 En este mismo sentido Kaplan de Drimer \& Drimer (1975) y Lambert (1961).

35 En él se traza la siguiente estrategia para conseguir cooperativizar la economía mundial: partiendo inicialmente del establecimiento de una tienda para víveres, ropas, etc., se satisfarían las necesidades de consumo de los socios; seguidamente, se continuaría con la construcción de viviendas para los socios; posteriormente se iniciaría la fabricación de artículos y la compra de unos terrenos para ser cultivados, procurando empleo a los miembros sin ocupación; finalizando con el establecimiento de una colonia autosuficiente donde se organizaran las fuerzas de producción, distribución, educación y gobierno junto con el apoyo a otras sociedades para el establecimiento de colonias similares.

36 Si bien en una primera fase plantea un programa de transformación a través de la expansión de sus asociaciones hasta alcanzar la corporación del futuro, en una segunda fase posterior, abandona esta idea y considera que sus asociaciones tienen una importancia reducida dentro del sistema.

37 Como se verá en el siguiente epígrafe, las asociaciones de Schultze-Delitzsch y Raiffeisen terminaron abandonando la ACI en el congreso de Budapest de 1904 al no querer alinearse con los cooperadores de inspiración capitalista ni con los que admitían las ayudas del Estado. 
en proporción al capital social aportado, la responsabilidad ilimitada y solidaria de $\operatorname{los} \operatorname{socios}^{38}$ y la devolución del fondo de reserva a los asociados en caso de disolución.

También cabría destacar la gran influencia de Schultze-Delitzsch en la dotación de un código normativo a sus asociaciones cooperativas. Fue él mismo el que en 1863 presentó ante el Parlamento prusiano el proyecto normativo sobre cooperación y que finalmente terminaría cristalizando en la primera ley prusiana de cooperativas (1867), la cual, a su vez, ha ejercido una gran influencia en la vigente ley alemana de cooperativas (1889) (Vargas Vasserot, 2015). Asimismo, reseñar la importancia de la figura de este autor en la creación de la actual Deutscher Genossenschaftsverband (Federación de cooperativas alemanas) ${ }^{39}$.

Las asociaciones que propone Schultze-Delitzsch están ligadas a la preocupación por la comunidad, ya que, como él mismo indica, a través de la asociación "el individuo se prepara para trabajar por el bien general de la comunidad a la que pertenece" 40 (Mladenatz, 1969:84). Para poder desarrollar estas asociaciones, durante una etapa inicial, recurre a los donativos y los préstamos sin interés de personas acomodadas, trasladando la preocupación por la sociedad a las clases más pudientes. Pero pronto aprecia que la solución no es la beneficencia, ni la filantropía, ni la ayuda del Estado, sino el esfuerzo y la ayuda mutua de aquellas personas a las que van dirigidas estas asociaciones (Kaplan de Drimer \& Drimer, 1975).

Las cooperativas de crédito de Schultze-Delitzsch adecuaban los plazos y las condiciones de los créditos a las necesidades de la clase media urbana a la que servían. Como consecuencia de estas prácticas, además de mejorar las condiciones económicas de sus miembros, consiguieron reducir los porcentajes aplicables al tipo de interés vigentes en Alemania, beneficiando a la comunidad en su conjunto ${ }^{41}$.

\subsubsection{Friedrich Wilhelm Raiffeisen}

Friedrich Wilhelm Raiffeisen (1818-1888) fue el creador de las primeras cooperativas de crédito en las áreas rurales (Raiffeisenbanken). Concibe la cooperación como una herramienta tanto para la defensa de los agricultores frente a los usureros, como para educar a los pobres y lograr la conservación de la paz social y la salvación del orden existente. Sus teorías están marcadas por las fuertes creencias cristianas que profesa, así, para Raiffeisen la única asociación que es capaz de mejorar la sociedad, es la asociación de fundamento cristiano $^{42}$. Aunque inició su carrera con la creación de sociedades de socorros para la compra de trigo, patatas y bestias de labor ${ }^{43}$, pronto advirtió la necesidad de crédito de los agricultores. De manera que creó en Heddesford en 1854 la Heddesdorfer Darlehnskassen-Verein cuyo éxito propició la multiplicación de este modelo de cooperativas de crédito por otras localidades alemanas.

A pesar de desarrollar su labor en Alemania y ser coetáneo de Schultze, sus sociedades presentan grandes diferencias. Así, Raiffeisen, constituye inicialmente sociedades sin capital en las que se recurría a las aportaciones de ahorros de los miembros más ricos con la intención de que fuesen prestados a los miembros menos pudientes que necesitaban crédito. Apelando a las convicciones religiosas de los más ricos y a los sentimientos de amor al prójimo y solidaridad, consigue persuadirles para que aporten sus ahorros y adquirieran una responsabilidad ilimitada y solidaria, recibiendo estos a cambio únicamente el interés habitual y la exclusividad en la gestión de la cooperativa, aunque de manera no remunerada. Como consecuencia de esto último, los miembros de las cooperativas de Raiffeisen no ostentaban los mismos derechos ${ }^{44}$. Estas sociedades también se caracterizaban por realizar múltiples actividades, no solo concedían crédito, sino que también aprovisionaban suministros a sus miembros y distribuían sus productos, tenían un ámbito territorial limitado y exigían ciertas cualidades morales para la adhesión de nuevos miembros. Así pues, puede decirse que en esta primera etapa las cooperativas de Raiffeisen son más bien entidades de beneficencia entre cristianos con un marcado carácter filantrópico.

Con el paso del tiempo las características de estas entidades evolucionaron. En concreto, a raíz de la aprobación de la Ley de cooperativas promovida por Schulze, las cooperativas de Raiffeisen debieron pasar a estar constituidas con un capital formado por suscripciones de todos sus afiliados, tanto de los ricos, como de

Aunque, tras la aprobación de la Ley de cooperativas prusiana de 1867, la responsabilidad pasaría a ser limitada.

39 Schultze-Delitzsch fue el promotor del primer congreso de cooperativas de crédito del modelo Schultze celebrado en Weimar en 1859 , en el que se decidió la creación de una Oficina Central (Zentral-Korrespondenzbureau), la cual quedó bajo la dirección de Schultze-Delitzsch hasta su muerte, y que posteriormente terminó transformándose en dicha federación.

40 Texto extraído de la carta que Schultze dirige al profesor italiano Francesco Viganò.

41 Recoge Mladenatz (1969) que en 1889 la cooperativa Blatter fúr Genossenschaftswesen, fundada por Schulze-Delitzsch, publicó la variación del tipo de interés entre 1862 y 1887 en varias regiones alemanas. Así, en Prusia disminuyó del 10.22\% al 6\% y en Baden del 7.98\% al 5.56\%.

42 Como consecuencia de esto, sus entidades estarán formadas únicamente por miembros católicos y protestantes (Lambert, 1961).

43 Vargas Vasserot (2015:59) las define como "sociedades precooperativas de carácter caritativo".

44 Como expresa Lambert, en las cooperativas de Raiffeissen encontramos dos clases diferenciadas: los ricos, que son al mismo tiempo los gobernantes y maestros, y los pobres, que son al mismo tiempo los gobernados y los educandos. Raiffeisen justifica esta forma de distribuir la gestión de la siguiente manera: "corresponde en primer lugar a los ricos, por su entrada en la cooperativa, por su responsabilidad que compromete toda su fortuna por tomar a su cargo la dirección de la empresa gratuitamente, ya que renuncian tanto a cualquier remuneración como a cualquier dividendo y sus aportaciones no reciben más que el interés normal, la misión de colaborar de la forma más altruista posible y al mismo tiempo de ejercer una influencia sobre los miembros pobres, por la palabra y el ejemplo, despertando en ellos el celo necesario para que se eleven por sí mismos" (Lambert, 1961:103). 
los pobres. Además, la importancia que tenían las aportaciones de los ricos comenzó a reducirse y las entidades de Raiffeisen se transformaron paulatinamente en entidades de ayuda mutua de todos los socios.

La búsqueda del beneficio de la comunidad en las organizaciones de Raiffeisen se muestra tanto desde un momento inicial, cuando trata de eliminar la usura en el comercio de bestias de labor, como posteriormente, cuando para satisfacer las necesidades de crédito de los más pobres, consigue convencer a los más ricos a comprometer toda su fortuna de manera solidaria. Este interés por la comunidad fue conservado por las organizaciones de crédito creadas por Raiffeisen al transformarse en asociaciones de ayuda mutua. Así, los estatutos debían fijar que la sociedad no era creada para obtener beneficios, por lo que los excedentes que se lograsen debían ir destinados a fondos de reserva y a obras sociales. Igualmente, en caso de disolución de la entidad, los fondos no podían ser distribuidos entre los socios, sino que debían ser entregados a otra asociación. También cabe señalar la reducida cuantía de las participaciones sociales necesarias para adherirse a la entidad, de unos pocos marcos o unos pocos peniques, lo cual facilitó que una gran cantidad de población pudiera ingresar en la cooperativa y se beneficiara de las ventajas de la misma.

\subsubsection{Adaptación de los modelos precedentes a las condiciones del país: Luzzati y Desjardins}

Los sistemas de Schulze-Delitzsch y Raiffeisen, tras realizarles algunas modificaciones, fueron implantados en otros países dentro del movimiento cooperativo rural, así como en el de las clases medias de las ciudades (Mladenatz, 1969). Ejemplo de esto son los casos en Italia del cooperativismo desarrollado por Luzzatti y en Canadá, el caso de Desjardins.

Luigi Luzzatti (1841-1927) fue el introductor del cooperativismo de crédito en Italia ${ }^{45}$. Los bancos populares de Luzzatti modificaron el sistema de Schulze-Delitzsch para adaptarlo a las particulares condiciones económico-sociales italianas. Así, sus cooperativas tenían una responsabilidad limitada a las acciones suscritas por los socios, el control y la administración de la entidad eran ejercidos por los propios socios, los cargos no eran remunerados, dotó de una mayor importancia a los fondos de reserva llegando a alcanzar un valor superior al capital social y, a diferencia del modelo alemán, admite la ayuda estatal. Cabría destacar la autoría de una frase que define su pensamiento cooperativista: "la caja de ahorro es el dinero del pobre prestado al rico, mientras que la cooperativa, al contrario, es el dinero del pobre prestado al pobre" (Kaplan de Drimer \& Drimer, 1975:254). Entre las actuaciones de los bancos populares de Luzzatti en las que se aprecia una preocupación por la comunidad se encuentra la concesión de préstamos a los más pobres que no generan intereses y sin necesidad de más garantía que su palabra de honor. Además, tuvo en cuenta el nivel económico de la población italiana y estableció unas participaciones sociales de cuantía muy reducidas en comparación con las que exigía Schulze-Delitzsch (de entre 25 y 50 liras, e incluso en ocasiones reduciéndose hasta las 5 liras), lo que facilitó la adhesión y la participación en sus cooperativas y, por consiguiente, favoreció el acceso al crédito a un mayor número de personas.

Por su parte, Alphonse Desjardins (1824-1920) es el precursor del cooperativismo de crédito en Canadá. Tras pasar varios años en Europa estudiando las cooperativas de crédito, trasladó y adaptó los sistemas de Schulze-Delitzsch y Raiffeisen a Canadá. Así, en 1900 fundó en Quebec la primera de sus credit-unions, basadas en la adhesión voluntaria, la obligatoriedad de los socios de suscribir acciones sociales, la responsabilidad limitada, y la distribución de excedentes en proporción a las acciones cooperativas ${ }^{46}$. La intención que subyace detrás de la creación de estas entidades está ligada a una intensa preocupación por la comunidad, ya que la finalidad de las mismas, según recoge Benevides Pinho (1987:57) era "conducir a los campesinos, obreros y artesanos a la responsabilidad de la autodirección de sus propios destinos, sobre bases democráticas, a la autoprotección frente a las tasas usurarias de interés y los abusos del crédito, así como al compromiso consciente con el progreso social".

\section{La ACI y el principio de interés por la comunidad}

Una vez alcanzado el final del siglo XIX la expansión del movimiento cooperativo es una realidad, aunque no se puede decir que se trate de un movimiento unívoco en el que se defiendan y promuevan las mismas ideas cooperativas, sino que, tal y como se ha visto, la idea de qué es una cooperativa y cómo debe funcionar varía dependiendo del país y de la escuela que la aborde, coexistiendo múltiples doctrinas cooperativas a lo largo del mundo. Además, al igual que ocurre con el contexto socioeconómico y geopolítico, la teorización cooperativa defendida por decenas de autores diferentes, irá variando en el curso del siglo XX, generando a

45 En 1864 fundó el primer banco popular en Lodi, y al año siguiente hizo lo propio con el de Milán. Este tipo de cooperativa se popularizó rápidamente, llegando a alcanzar en 1890 hasta 624 entidades repartidas por toda la geografía italiana (Monzón Campos, 1989). Para una mayor información acerca de la vida como profesor universitario y político de Luzzati se puede consultar obras como Divar Garteiz-Aurrecoa, J. (2012) Luigi Luzzatti, "pionero del crédito popular».

46 El modelo cooperativo de Desjardins se trasladó rápidamente a Estados Unidos tras colaborar junto con Filene y Bergengren en la redacción de la primera ley reguladora de credit-unions en 1909. 
su vez modificaciones en la interpretación y la importancia del interés por la comunidad en el seno cooperativo. Como es evidente, me es imposible recoger aquí las ideas de cada uno de ellos y analizar su relación con el principio de interés por la comunidad ${ }^{47}$. Por ello, nos centraremos exclusivamente en analizar la evolución que ha sufrido el interés por la comunidad en el seno de la ACI, por ser la "guardiana de los valores y principios cooperativos" y la principal defensora del "modelo económico empresarial de las cooperativas, basado en valores específicos" (art. 1 Reglamento ACI), sin perjuicio de que se pueda hacer referencia a alguno de esos autores a lo largo del texto, bien porque se considere necesario para entender más adecuadamente algún cambio de paradigma en el interior de la ACI, o bien porque teoricen sobre algún aspecto que influye directamente sobre el principio de interés por la comunidad y se considere oportuno.

\subsection{El interés por la comunidad en el origen de la ACI}

La ACI, al igual que el movimiento cooperativo, no surge de una forma espontánea. Anteriormente a que este organismo se creara como tal, ya se habían desarrollado múltiples organizaciones internacionales que asociaban y promovían el movimiento cooperativo ${ }^{48}$. Todas estas experiencias previas pusieron los cimientos para que entre los días 19 y 24 de agosto 1895, en el Primer Congreso Internacional Cooperativo de Londres, se creara la Alianza Cooperativa Internacional. En sus primeros años de vida, la ACI da muestra de cierta preocupación por unos intereses distintos a los de los socios al incluir en el artículo 1 de sus estatutos, entre los objetivos de la cooperación, la aceleración de la participación de toda clase de personal en los beneficios de las cooperativas, independientemente del tipo de cooperativa que sea $^{49}$. La inclusión de esta cláusula en el estatuto se debe a la mayor influencia que ejercieron los partidarios de las cooperativas obreras de producción sobre los defensores de las cooperativas de consumo ${ }^{50}$.

Como se puede deducir de los debates llevados a cabo en estos dos primeros congresos, los primeros años de la ACI son años convulsos ${ }^{51}$. Aunque inicialmente la ACI adopta una tendencia cercana a la promoción de las cooperativas de producción, esta situación varía a partir del Congreso de Manchester de 1902 en el que se elimina la posibilidad de adhesión a la ACI a personas físicas. Esta decisión, con una clara intención de apartar de la Alianza a los miembros individuales franceses e ingleses ${ }^{52}$, consigue desplazar a la corriente partidaria de la participación en beneficios e instaurar el dominio de las cooperativas de consumo, el cual se verá aún más fortalecido tras el siguiente Congreso, el de Budapest de 1904, en el que se evidencian ciertos desacuerdos entre los miembros de la ACI, e incluso se produce la salida de algunas organizaciones cooperativas $^{53}$. Así, tras este Congreso de 1904, puede decirse que la ACI pasa a estar dominada por las cooperativas de consumo ${ }^{54}$.

47 La exposición pormenorizada de las distintas doctrinas cooperativas ha sido realizada por voces autorizadas como Lambert (1961), Kaplan de Drimer \& Drimer (1975), Mladenatz (1969), Totomiaz en sus obras La cooperazione y Pantheon cooperativo, o Desroche en Il progetto cooperativo.

48 En este sentido, se puede mencionar como la precursora de este tipo de entidades a la Association of all Classes of all Nation creada por Owen en 1835, la cual únicamente acogió en su seno a miembros de Inglaterra y no tuvo el éxito esperado. A esta experiencia le siguieron varios congresos organizados principalmente por las cooperativas inglesas en los que también participaron delegados de otros países, como por ejemplo el Congreso de la cooperación inglesa celebrado en Londres en 1869, a la que acudieron 18 delegados extranjeros, o el Congreso Cooperativo de Plymouth de 1886, donde se volvió a retomar la idea de constituir una organización internacional de la cooperación, tras la propuesta realizada por Emile de Boyve de fundar una liga internacional entre los cooperadores franceses, italianos e ingleses. Esta idea tuvo que esperar a hacerse realidad hasta 1892, cuando se crea en Londres la primera organización internacional, la Asociación de Amigos de la Cooperación de Producción, presidida por Greening y en la que también participaron otros ilustres del cooperativismo de producción como Vansittart Neale, Leclaire, Godin y Robert. Esta asociación promulgaba el plan de emancipación del productor, defendiendo la participación del trabajador en los resultados como el principal criterio de autenticidad cooperativista. Estas ideas eran completamente opuestas a la postura defendida por el grupo que apoyaba la hegemonía del consumidor, entre los que se encontraban Gide y la escuela de Nimes (Benevides Pinho, 1987).

49 El contenido de dicho artículo decía textualmente: "Apresurar, por todos los medios de propaganda a su alcance, - la participación del personal en los beneficios siendo el propio de cualquier cooperación, - el momento donde todas las Asociaciones que lleven el nombre de Cooperativas, ya sean industriales, agrícolas, de consumo, de crédito o de construcción, habrán organizado, en favor del trabajo, la participación en los beneficios extendida a todo su personal sin excepción y habrán incluido en sus estatutos la obligación de practicar dicha participación en los beneficios" (art. 1.3 Estatutos de la ACI) (ACI, 1897:152).

50 En el anterior Congreso de 1895 ya se había producido el primer enfrentamiento entre los defensores de ambas posturas, llegando a debatir intensamente a causa de la inclusión de la participación en los beneficios entre los principios de la cooperación (ACI, 1895). Finalmente, consiguieron que se incluyera dicha postura en los estatutos, aunque sin llegar a exigir para la admisión en la ACI que dicha participación estuviera incluida en los estatutos, lo que hubiera provocado la salida de las cooperativas inglesas de consumo de la ACI (Mladenatz, 1969).

51 La historia de la ACI durante estos primeros años de creación ha sido dividida por diversos autores en tres etapas, aunque cada uno de ellos ha establecido diferentes periodos. Así Gide sitúa en primer lugar la etapa burguesa, la cual se desarrolla entre los Congresos de 1897 y 1902 y se caracteriza por el dominio de la participación en beneficios. La siguiente etapa que se desarrolla entre los Congresos de 1904 y 1907 la denomina como la etapa socialista. Y finamente la etapa autónoma o de la neutralidad es la que se produce a partir del Congreso de 1910, en la que se declara la neutralidad política de la ACI. En cambio, Mladenatz, coincide con Gide en la primera etapa, pero divide las siguientes en periodos diferentes, situando la segunda etapa entre los Congresos de 1902 y 1924 y caracterizada por la dominación de las cooperativas de consumo, y la tercera etapa a partir de 1924 caracterizada por la expansión de la concepción cooperativa, abarcando también a pequeños productores independientes (Mladenatz, 1969).

52 A esta conclusión llega Mladenatz (1969:129).

53 En dicho congreso, por un lado, chocaron las ideas de las cooperativas de consumo, que pretendían una reforma económico-social con tendencias anticapitalistas, con las ideas de las cooperativas de crédito de los sistemas de Schultze-Delitzsch y Raiffeisen, que eran partidarias del mantenimiento del sistema existente; mientras que por otro lado entró en conflicto la tesis que admitía la ayuda estatal, con las que la rechazaban por miedo a ver peligrar la autonomía de la cooperativa, posición defendida por las cooperativas de crédito. Finalmente prevalecieron las 
Esta asunción de poder por el movimiento de consumo provocó alteraciones en las escasas referencias a la preocupación por intereses distintos a los de los socios. Así, en la declaración de principios presentada por Hans Müller en el Congreso de Hamburgo de 1910 y que fue adoptada por la ACI, las aspiraciones del movimiento cooperativo cambian, pasando de intentar hacer partícipes a todos los trabajadores de los beneficios, a un objetivo casi utópico: la transformación del sistema capitalista a través de la organización de todos los consumidores en cooperativas de consumo ${ }^{55}$. Esto es lo que se ha venido a denominar la doctrina cooperativa de la soberanía del consumidor, la cual entiende que el interés de todos los consumidores asociados se confunde con el interés de toda la comunidad, es decir, con el interés general, y en la que Gide fue uno de sus máximos exponentes ${ }^{56}$. Por último, también hay que indicar que en esta misma declaración la ACI no descuida los intereses de los trabajadores no socios, ya que reconoce que las cooperativas, como empleadoras, siguen teniendo el deber de "acordar a sus empleados y obreros condiciones de trabajo y salarios modelo y reconocerles también su derecho de asociarse" (Mladenatz, 1969:137). Con esta declaración se pone de manifiesto el nuevo dominio de las cooperativas de consumo en la ACI.

Esas mismas ideas de sustitución del sistema capitalista son mantenidas durante los años posteriores, tal y como se evidencia de la modificación realizada en el artículo 1 de los estatutos de la ACI durante el Congreso de Basilea de 1921, en el que se incluye que la ACI persigue "la sustitución del régimen de empresas privadas con fines de lucro por un régimen cooperativo organizado en interés del conjunto de la comunidad y basado sobre la ayuda mutua" (ACI, 1921:72). En ese mismo congreso, Serwy propone un proyecto de resolución en el cual se les pide a los sindicatos que consideren a las cooperativas "como de carácter anticapitalista y de lucha en favor de la comunidad" y vuelve a incidir en la preocupación de las cooperativas por las condiciones laborales de los trabajadores al afirmar que las cooperativas "se esfuerzan para acordar a su personal las mejores condiciones de trabajo posibles, que aceptan los contratos colectivos de trabajo" (Mladenatz, 1969:140).

\subsection{La ausencia de este principio en la primera versión de los principios cooperativos (1937)}

A pesar de que los estatutos de la ACI adoptados en 1896, en su artículo 1, ya fijaban entre los objetivos de la organización la difusión de los verdaderos principios cooperativos (ACI, 1897), hay que esperar hasta el Congreso de Estocolmo de 1927 para encontrar la primera referencia a la necesidad de establecer formalmente unos principios comunes para todas las cooperativas. En este congreso fue presentado el informe de Albin Johansson, director de la Unión de Cooperativas Suecas, titulado Problems of Modern Cooperation, en el que mostró las dificultades que encontraban las cooperativas a la hora de aplicar e interpretar el programa de Rochdale, debido a que algunas de esas reglas no se adaptaban a los tiempos cambiantes ni a la expansión del movimiento cooperativo del momento y provocando que las cooperativas las incumplieran (Johansson, 1927). A esta postura se adhirió, tres años después, en el Congreso de Viena de 1930, la Federación Nacional Francesa de Cooperativas de Consumo con su memorándum titulado Los Principios de Rochdale, que exponía que "en la práctica, según las circunstancias y los lugares, los principios de Rochdale se han interpretado de manera muy diversa", por lo que solicitaron la designación de un Comité Especial para "investigar las condiciones en las que se aplican los principios de Rochdale en varios países y, si es necesario, definirlos" (ACI, 1930:156). Esta propuesta fue adoptada tras un debate en el que se enfrentaron 3 posturas diferentes ${ }^{57}$, dentro de las cuales, por su relación con el interés por la comunidad, nos

primeras posturas expuestas, lo que provocó la salida de la ACI de las cooperativas del tipo Schultze-Delitzsch y Raiffeisen y de las organizaciones cooperativas agrícolas, que posteriormente fundarían su propia asociación internacional bajo el impulso de Haas y von Störk.

54 En el interior de la ACI comienzan a su vez a dominar las ideas de los socialistas cristianos ingleses, de Gide y de la Escuela de Nimes (Monzón Campos, 1989)

55 En dicha declaración se fija un programa cooperativo para alcanzar la sustitución del sistema capitalista. Este se iniciaría mediante organización de las cooperativas de consumo, lo cual permitiría, gracias a las rentas obtenidas de esa organización, pasar a organizar la producción sobre la base cooperativa. En ella se establece que la cooperación "tiene por fin la salvaguarda de los intereses del trabajo" y "todas las formas de cooperación tienden hacia una distribución más equitativa de la riqueza social en favor de las clases laboriosas". Sin embargo, "las sociedades cooperativas de consumo constituyen la categoría más importante, no sólo porque ellas representan los intereses más amplios y más generales del pueblo trabajador, sino [..] sobre todo, por los principios económicos que se hallan en su base, principios que, para su aplicación y extensión en la realidad, no tienden sino a una trasformación del sistema capitalista".[..] "En la medida en que los consumidores se agrupen en las cooperativas de consumo, están creando, por ese hecho, una organización con la fuerza de compra de la renta del trabajo, organización que coloca a las clases laboriosas en condiciones de organizar, cada vez con mayor amplitud, el propio trabajo sobre la base cooperativa". (Mladenatz, 1969:134-136)

56 Esta doctrina tiene su origen en las ideas de King y Derrion, las cuales fueron posteriormente desarrolladas con una mayor amplitud en Alemania e Inglaterra, con autores como Pfeiffer, Mitchell o Potter. Sin embargo, fue Gide, en Francia, el que la expuso de una manera más brillante (Lambert, 1961). Es imposible abarcar aquí toda la doctrina desarrollada por Gide y exponer el importante papel desempeñado por sus ideas en el cooperativismo de consumo. De una manera muy resumida, cabría señalar su programa de transformación pacífica de la sociedad hasta alcanzar, lo que él llamó, la República cooperativa, en la que los consumidores, asociados en cooperativas, organizarían todas las actividades económicas, complacerían todas sus necesidades y conseguirían eliminar el lucro. Para ello establece un plan de tres etapas: en la primera se conquistaría el comercio a través de cooperativas de consumo, en la segunda, con los capitales conseguidos en la primera, el movimiento cooperativo conquistaría la industria y en la tercera, se conquistaría la agricultura.

57 Entre las otras posturas que aparecieron en el debate se encontraban los que afirmaban que era inviable aplicar unas reglas que tienen cien años de antigüedad para tratar de resolver necesidades socioeconómicas actuales y abogaban por alcanzar un acuerdo sobre la interpretación actual de 
interesa destacar la de los soviéticos. Estos propusieron eliminar el principio de distribución de beneficios en proporción a las compras, al considerarlo un principio puramente capitalista, y, en su lugar, destinar los beneficios a "la consolidación de la posición financiera de las Sociedades de Consumidores, la construcción de nuevos edificios, la labor cultural y educativa para elevar el nivel de vida, respetando el principio de clase" (ACI, 1930:159).

Cuatro años después se celebró el Congreso de Londres de 1934, donde el Comité especial presentó por primera vez una lista de "siete puntos que representan los Principios esenciales de Rochdale, para cada uno de los cuales la justificación se encuentra en la constitución, reglas y práctica de la Sociedad original" (ACI, 1934:134). Esos primeros siete principios eran: la adhesión libre, el control democrático, los dividendos sobre las compras, el interés limitado sobre el capital, la neutralidad política y religiosa, las ventas al contado y la promoción de la educación ${ }^{58}$. Como puede observarse, varios de estos principios están referidos exclusivamente a cooperativas de consumo ${ }^{59}$, esto es consecuencia, entre otras razones ${ }^{60}$, de la composición de la ACI, con una mayoría de miembros pertenecientes a este tipo de cooperativas ${ }^{61}$. Tras un debate agitado no consiguió aprobarse el informe con la propuesta, por lo que se acordó seguir estudiando el asunto y posponer el acuerdo para el siguiente congreso. De forma que en el Congreso de París de 1937, tras realizar unas nuevas encuestas adaptadas a los grupos de cooperativas que no pertenecían a las cooperativas de consumo, se presentó un informe casi idéntico al de 1934, con los mismos siete principios, el cual fue finalmente aprobado casi por unanimidad, votando a favor todos los representantes excepto dos (ACI, 1938).

Debemos destacar que en esta primera versión de los principios cooperativos no hay ninguna referencia expresa a un principio independiente que reconozca el interés por la comunidad, a pesar de ser una de las actitudes que sí tomaban en consideración sus predecesores y, en concreto, la cooperativa de Rochdale había llegado a incluir algunas de esas actuaciones en sus estatutos. Tampoco se tuvo en cuenta la propuesta realizada por los soviéticos de destinar los beneficios a causas sociales, ya que finalmente entre los principios se incluyó la regla de los dividendos sobre las compras. Incluso llama la atención que a lo largo del informe no se realice ninguna apreciación acerca de la mejora de las condiciones laborales de los trabajadores no socios, cuando el tema había sido abordado en varios de los congresos precedentes.

Lo más cercano a un posible reconocimiento oficial de dicho interés lo encontramos en parte del contenido de los informes de 1934 y 1937. En ellos, además de la propuesta de principios cooperativos, se indicaba que, a partir de las encuestas enviadas a las organizaciones cooperativas, se habían detectado cuatro prácticas que habitualmente realizaban las cooperativas, pero que su categorización como principio era controvertido $^{62}$. Precisamente es en una de esas prácticas que no alcanzan la categoría de principio donde encontramos una muestra de la preocupación de las cooperativas por los intereses de la comunidad, al adjudicar, en caso de liquidación, el fondo de reserva inalienable a otra cooperativa o "a alguna otra organización desinteresada de utilidad pública" (ACI, 1934:156), "para ayudar a las cooperativas que luchan contra las dificultades o para que se utilice para obras de bienestar social, educativas o de utilidad pública" (ACI, 1938:183) en lugar de distribuirlo entre los socios ${ }^{63}$. Hay que señalar que esta conducta ya había sido identificada previamente en el Congreso de Gante de 1924 por Albert Thomas como una de las 8 características que definen y distinguen a las cooperativas ${ }^{64}$. Pero volviendo a los informes, para el Comité

los mismos, representados por Cleuet; y los que consideraban que los principios se encuentran definidos en el artículo 8 de los estatutos de la ACI y no era necesario revisarlos, representados por Worley (ACI, 1930).

58 Estos principios, a su vez, fueron divididos en dos categorías, los cuatro primeros conforman el grupo de los que son identificados como obligatorios y, según menciona el Secretario General de la ACI durante el debate sobre el informe, conforman la base económica de la cooperación y representan un nuevo régimen económico que es capaz de reemplazar el orden capitalista existente; mientras que los tres últimos son considerados como métodos de acción y de organización (ACI, 1934).

59 En este mismo sentido se pronuncia Mateo Blanco (1985).

60 La propuesta de 1934 presentó varias deficiencias en su elaboración. En primer lugar, para la confección de la encuesta solo se analizaron los primeros estatutos de los Pioneros de 1844, obviando las posteriores modificaciones, por lo que dejaron fuera algunas incorporaciones posteriores como las reglas de la división del activo en caso de disolución de la sociedad y la referencia al comercio con los no socios (en este mismo sentido Lambert (1961). Y en segundo lugar, resulta extraña la forma en que se realizaron las encuestas. Y es que de las 47 organizaciones a las que se les envió la encuesta, 28 eran cooperativas de consumo, más de la mitad. Así lo llega a reconocer la ACI en el posterior Congreso de 1937, diciendo que el informe presentado en 1934 investigaba la aplicación actual de dichos principios por las cooperativas de consumo (ACI, 1938).

61 Aunque este hecho se ha puesto de manifiesto en el epígrafe anterior al hablar del dominio de las cooperativas de consumo, hay que señalar que la ACI llega a reconocerlo expresamente en el informe de la Comisión sobre los Principios cooperativos de 1966, al admitir que "el Comité especial de la ACI pudo haber estado influenciado por la composición de la Alianza en aquella época” (ACI, 1975:32), con una mayoría formada por cooperativas de consumo.

62 Estas prácticas eran el comercio exclusivo con miembros, la voluntariedad en la cooperación, la venta al precio corriente o de mercado y la indivisibilidad del activo neto y la adjudicación del fondo de reserva inalienable. Según el informe de 1934, las dos primeras prácticas podrían alcanzar la misma importancia que los principios cooperativos, mientras que de las otras dos no podía decirse que fuesen esenciales para la definición del sistema rochdaliano (ACI, 1934).

63 Como se vio anteriormente, esta conducta fue incluida en los estatutos de Rochdale tras la modificación de 1854 , pero al elaborar las encuestas basándose exclusivamente en los estatutos de 1844 obviaron su incorporación.

64 En dicho congreso Thomas presentó un informe elaborado junto con Fauquet, titulado The Relation between the Different Forms of Co-operation, en el que dedica el primer apartado al establecimiento de las características comunes y distintivas de las cooperativas. Una de las 8 características que definen a las cooperativas, hace referencia a la distribución del activo sobrante en los casos de disolución de las cooperativas. En ella señala que había observado que este se distribuía según tres métodos diferentes: se reparte en partes iguales; se distribuye según la misma regla que se 
especial esta práctica ni siquiera era esencial para la definición del sistema rochdaliano, por lo que únicamente recomienda que sea considerada favorablemente por los movimientos nacionales con vistas a su incorporación en las diferentes legislaciones cooperativas estatales (ACI, 1934) ${ }^{65}$. Esta misma postura es la que adopta Fauquet en el debate posterior, haciendo un llamamiento a las organizaciones cooperativas a que presionen para que sus legislaciones incorporen dicha cláusula ${ }^{66}$ y defendiendo la obligación que tienen las cooperativas de aplicar desinteresadamente estas reservas.

También se aprecian en estos informes otras prácticas que denotan cierta preocupación por la comunidad, como era la devolución de la mitad de los beneficios sobre las compras a los no miembros, aunque se señala que dicha práctica era realizada por muy pocas de las organizaciones encuestadas (ACI, 1934); o la alusión a que la gran reivindicación del movimiento cooperativo era instaurar un nuevo régimen económico basado en los principios básicos, "capaz de reemplazar el sistema capitalista competitivo existente” (ACI, 1934:160).

A pesar de estas breves referencias, no puede decirse que la ACI en estos primeros principios tenga presente la defensa de unos intereses distintos a los de los socios.

\subsection{La referencia al servicio de los intereses de la comunidad en la segunda versión de los principios cooperativos (1966)}

A los pocos años de la celebración del Congreso de París, estalla la segunda guerra mundial, un suceso que provocó grandes alteraciones políticas y económicas en todo el planeta. Como es obvio, el movimiento cooperativista también se vio afectado por todos esos $\operatorname{cambios}^{67}$, aunque en el marco de la ACI se mantuvo el dominio del cooperativismo de consumo. Sin embargo, uno de los hechos reseñables con respecto al principio de preocupación por la comunidad, se enmarca precisamente fuera de las actuaciones de la ACI. En 1959 se publica la obra de Lambert titulada La doctrina cooperativa, uno de los primeros estudios en los que se le reconoce al interés por la comunidad la categoría de principio cooperativo. En ella Lambert realiza la siguiente definición de la sociedad cooperativa, de la que nos interesa especialmente la última parte: "Una sociedad cooperativa es una empresa constituida y dirigida por una asociación de usuarios, que aplica en su seno la regla de la democracia y que tiende directamente al servicio tanto de sus miembros como del conjunto de la comunidad" (Lambert, 1961:261). Para este autor, la cooperativa debe estar al servicio de sus miembros pero "sólo en la medida en que es legítimo y compatible con el interés general" (Lambert, 1961:266), y niega que este interés por el conjunto de la comunidad sea un principio nuevo para las cooperativas, sino que también estaba incluido en las cooperativas de King, Buchez, Raiffeissen e incluso en Rochdale. Con respecto a esta última, Lambert identifica el interés por la comunidad en el capítulo segundo de su obra ${ }^{68}$, tras realizar un nuevo análisis de los estatutos originales de Rochdale y sus posteriores modificaciones, concluye que él añadiría tres principios más, de los cuales nos interesa especialmente uno de ellos $^{69}$, el que establece que la actividad de las cooperativas es una "actividad dirigida a servir al interés de los miembros, pero sólo en la medida en que este interés se juzga conforme al interés general de la comunidad (espíritu de servicio)" (Lambert, 1961:57), un interés general que identifica "con el interés de la inmensa mayoría de los ciudadanos" (Lambert, 1961:82). Así pues, en esta obra Lambert reconoce el carácter de interés por la comunidad entre las prácticas cooperativas, al cual él llama espíritu de servicio, analiza la importancia de dicho carácter dentro de los elementos que definen a las cooperativas, le otorga

sigue en la distribución de los beneficios; "o mejor aún, se transfieren a otra organización cooperativa o a una institución pública" (Thomas, 1924:100). Con esta última referencia Thomas mostraba una clara preferencia por la última opción.

65 Kaplan de Drimer \& Drimer (1975:148) consideran que esta norma no fue adoptada como principio ni en 1937 ni en 1966 debido a "razones prácticas impuestas por la diversidad de posiciones existentes".

66 Tal y como dice Fauquet, en Francia ya habían conseguido que dicha obligación fuese exigida por la legislación cooperativa tras una petición realizada por las distintas organizaciones cooperativas y su federación, por lo que proponía que, en aquellos países en los que la legislación impusiese un fin diferente a la aplicación desinteresada del activo, las organizaciones cooperativas de esos países hiciesen el esfuerzo necesario para aproximar la legislación en este sentido (ACI, 1934).

67 Entre los efectos provocados en el cooperativismo podría destacarse la expansión del mismo. En este sentido, algunos de los países que habían sido devastados por la guerra, como fue el caso de Japón, recurrieron al cooperativismo para reactivar su economía. Además, la descolonización acontecida en países africanos y asiáticos también propició la expansión del cooperativismo a países como India. Por otro lado, la segunda guerra mundial también ocasionó la asunción por Rusia del poder sobre un variado número de países europeos de tradición cooperativa, con la consecuente influencia del sistema político y económico comunista sobre las cooperativas existentes. En estos países terminó imponiéndose la cooperación agrícola y provocando a su vez la salida de esos países de dirigentes y expertos cooperativistas, y la censura y persecución de algunas de sus obras, como le ocurrió a Mladenatz. Sobre las consecuencias generadas en el movimiento cooperativo por la segunda guerra mundial puede verse a Mateo Blanco (1985).

68 Lambert lo titula "Nuevo análisis de los principios de Rochdale y, en general, de los principios cooperativos".

69 Los otros dos principios que añade Lambert también tienen que ver con la preocupación por la comunidad, sin embargo, el que recogemos en el texto es el principio por la comunidad tal y como es entendido en la actualidad. Los otros dos principios, de los cuales Lambert indica que incluso se encuentran explícitamente contenidos en los estatutos de los Pioneros, eran la devolución desinteresada del activo neto en caso de disolución de la sociedad; y la aspiración a conquistar y cooperativizar la organización económica y social del mundo (Lambert, 1961). 
inicialmente ${ }^{70}$ la consideración de principio propio e independiente, e incluso decide incluirlo dentro de la definición que propone de cooperativa. Todo ello en 1959, treinta y seis años antes de que lo hiciera la ACI.

Durante estos años, en los congresos internacionales de la ACI se adoptan algunas resoluciones que muestran la preocupación del movimiento cooperativo por el futuro del planeta y sus comunidades. Esto se evidencia en algunas de las resoluciones del Comité Central, como la del Congreso de 1963, en la que se expresa que uno de los mayores problemas actuales es el mantenimiento de la paz y la seguridad, la prevención de una guerra termonuclear y el desarme de todos los países (ACI, 1963). Asimismo, las políticas de asistencia a países en desarrollo efectuadas por la ACI durante estos años ${ }^{71}$, revelan la preocupación del movimiento cooperativo por otras comunidades, a la vez que favorecen la expansión del cooperativismo.

Resulta incuestionable que los hechos acontecidos en los veinticinco años que habían transcurrido desde el Congreso de París tuvieron efectos tanto en las políticas y economías mundiales, como en la forma de actuar de las cooperativas, provocando que, de nuevo, los principios cooperativos perdieran parte de su eficacia. Este hecho fue puesto en evidencia en el Congreso de Bournemouth de 1963 por Klimov, cooperativista ruso representante de Centrosoyuz, el cual propuso llevar a cabo la revisión de los principios cooperativos tras exponer algunas de las prácticas que había observado en las cooperativas relacionadas con el cumplimiento de los principios ${ }^{72}$, entendiendo, además, que los principios ya no eran universales, sino que únicamente se adecuaban al cooperativismo capitalista ${ }^{73}$ (Miranda, 2014). Esta propuesta fue acogida de formas diferentes entre los asambleístas ${ }^{74}$, pero finalmente se adoptó la resolución por la cual se constituía una Comisión para analizar si las cooperativas respetaban en la práctica los principios de 1937 y, ante los resultados de dicho análisis, considerar si los principios debían de mantenerse o ser reformulados ${ }^{75}$.

El informe que presenta la Comisión en el Congreso de Viena de $1966^{76}$, recomienda que los principios se reconfiguren en seis, actualizando algunos de ellos e incluyendo otros que no fueron recogidos en la anterior declaración. De forma que los nuevos principios serían: la adhesión libre, el control democrático, el interés limitado sobre el capital, la distribución de los resultados positivos evitando que un socio obtenga ganancias a expensas de otros, educación y cooperación entre cooperativas ${ }^{77}$.

Tal y como se observa en este informe, no se le llega a otorgar de manera expresa a la preocupación por la comunidad la categoría de principio independiente. En cambio, sí que encontramos una referencia a la defensa de los intereses de la comunidad dentro del sexto principio cuyo texto dice "las cooperativas, para servir mejor a los intereses de sus miembros y sus comunidades, deben colaborar por todos los medios con otras cooperativas a los niveles local, nacional e internacional" (ACI, 1975:82). Al expresar que las cooperativas tienen que servir tanto a sus miembros como a sus comunidades, se entiende que las cooperativas tienen el deber de satisfacer los intereses de ambos. Como formuló Lambert, debe existir una coincidencia entre los intereses cooperativos y los de la comunidad, o, al menos, la satisfacción de los intereses de los miembros no debería causar ningún perjuicio a la comunidad ni menoscabar sus intereses.

70 Sin embargo, posteriormente Lambert realiza una reagrupación de todos los principios y termina introduciéndolo dentro de las reglas relativas a la formación y reparto de los beneficios y a la suerte que correrá el activo (Lambert, 1961).

71 Tras el Congreso de París de 1954, a la vista del trabajo elaborado por Watkins sobre el desarrollo cooperativo en los países subdesarrollados, la ACI decidió crear un fondo de desarrollo para esos países. En el siguiente Congreso celebrado en Lausana en 1960, se continuó con esta labor, adoptando un programa de asistencia técnica a largo plazo para promover el comercio entre organizaciones cooperativas de países industrializados y en vías de desarrollo. Estas actuaciones influyeron en la posterior cristalización del principio de cooperación entre cooperativas en 1966 .

72 Entre las prácticas habituales de algunas cooperativas que identifica la delegación soviética se encontraban la venta por debajo del precio del mercado, el abandono de la venta al contado y la inexistencia de la neutralidad política y religiosa en general (Lambert, 1984).

73 Esta petición se realiza en un contexto difícil para la ACI. Tal y como se ha comentado previamente, Rusia impuso su poder en varios estados europeos, suprimiendo libertades de dichos países y ocasionando, a su vez como efecto colateral de esto, la expulsión de sus organizaciones como miembros de la ACI. Estos fueron los casos de Polonia, Albania, Hungría y Alemania del este. Ante esta delicada situación y para evitar el peligro de que la ACI terminara dividiéndose en bloques antagónicos, se decide realizar una revisión de los principios cooperativos dictados en 1937 que los readapte en lo posible para procurar la reincorporación de los estados expulsados (Lambert, 1984).

74 Un grupo creía que era preciso realizar dicha revisión; otro grupo se mostró en contra de la misma, pero sí que creía necesario realizar una encuesta para controlar el cumplimiento de los principios; y un tercer grupo que creía que únicamente era necesario modificar las formulaciones de los principios del 37 (Mateo Blanco, 1985).

75 En concreto la resolución dictaba que la Comisión tenía la tarea de "determinar en qué medida los Principios de Rochdale tal como fueron definidos en el Congreso de París de 1937 se observan en nuestros días y las razones, en su caso, en que no puede hacerse" y "considerar, a la luz de los resultados del estudio citado, si los principios responden a la necesidad del movimiento, teniendo en cuenta la situación económica, social y política actual, o si algunos de ellos deben ser reformulados con el fin de contribuir mejor a la realización de los objetivos y de las tareas del movimiento en sus diferentes sectores" (ACI, 1975:27-28).

76 Dicho informe difiere bastante del que se presentó en 1937. En esta ocasión las encuestas se realizan a más de cien entidades de carácter variado, entre las que se encuentran organizaciones afiliadas a la ACI, no afiliadas y personas de reconocido prestigio en el ámbito cooperativo, además de incluir en la Comisión a miembros de muy distintas opiniones, comprendiendo así un mayor número de puntos de vista diferentes. Todas estas circunstancias propiciaron que en su elaboración se alcanzara la unanimidad de todos los miembros de la comisión y que estos recomendaran que el trabajo fuese aprobado sin modificaciones.

77 A diferencia de la anterior declaración en la que se realiza una división de los principios en función de su importancia, en el informe se establece que los principios son elementos esenciales de igual importancia pertenecientes a un mismo sistema, y necesitan observarse integralmente (ACI, 1975). Además, esta declaración, a diferencia de la anterior, no realiza una formulación breve de los principios, sino que desarrolla cada uno de ellos, tratando de plasmar con una mayor exhaustividad todas las ideas que conforman cada uno de los principios enunciados. 
Por ello, podemos decir que estamos ante la primera referencia en una declaración de principios de la ACI, a la especial preocupación por la comunidad que han de mostrar las cooperativas en su toma de decisiones.

La inclusión de esta nueva preocupación por los intereses de la comunidad dentro de este principio tiene su fundamento en el cambio de paradigma que se produjo durante los años previos en el seno de la ACI, al abandonar la doctrina de la soberanía del consumidor y de la búsqueda de la comunidad integral, y en su lugar, adoptar la idea de sector cooperativo formulada años antes por Fauquet ${ }^{78}$. La Comisión reconoce que "en todos los tiempos, el elemento común ha sido el hecho de que los mejores propósitos de la cooperación van más allá de promover simplemente los intereses de sus socios individuales. Su finalidad es más bien promover el progreso y el bienestar de la humanidad" (ACI, 1975:34), es decir, el elemento común de ambas doctrinas cooperativas y del movimiento cooperativo a lo largo de su historia ha sido la búsqueda de una mejora para la comunidad en su conjunto. Y en este camino para alcanzar "la meta más importante del Movimiento Cooperativo" como es "la promoción de los derechos económicos y sociales del pueblo" se requieren "esfuerzos concertados y activos hacia la realización de la paz mundial" (ACI, 1975:86). Por esta razón la Comisión decide situar el interés por la comunidad comprendido en el principio de intercooperación.

En el debate del Congreso se propusieron varias enmiendas a los principios propuestos, pero ninguna que afectara directamente a la formulación del principio de cooperación entre cooperativas, de forma que la breve referencia al interés de la comunidad no se vio alterada ${ }^{79}$. Por lo que finalmente se decidió la aprobación de los seis principios propuestos ${ }^{80}$.

\subsection{Etapa de sensibilización ante nuevos problemas sociales (1976-1992)}

Antes de comenzar con la siguiente etapa en la centenaria vida de la ACI, resulta de interés destacar la obra de Kaplan de Drimer \& Drimer titulada Las Cooperativas: fundamentos, historia, doctrina. En dicho trabajo hacen referencia a la coincidencia con los intereses generales de la comunidad en algunas actuaciones de los Pioneros de Rochdale, así como la inclusión de esa coincidencia de intereses en la reformulación de los principios cooperativos de 1966 en los que "si bien la ACI no llegó a consagrar dicha norma en un principio específico, la señaló en relación al sexto principio" (Kaplan de Drimer \& Drimer, 1975:577). Y de una manera casi premonitoria, recogieron que este interés por la comunidad terminaría siendo reconocido finalmente por la ACI en términos más explícitos y generales.

Retomando el tratamiento de dicho principio por la ACI, puede decirse que tras el Congreso de París de 1966 ya se habían colocado los cimientos para que se le reconociera a la preocupación de las cooperativas por los intereses de la comunidad la categoría de principio. Sin embargo, este hecho no tendrá lugar hasta el congreso de 1995. En el lapso de tiempo entre ambos eventos, las reuniones y congresos de la ACI se centraron principalmente en dos temas relacionados tanto con los intereses de la comunidad, como con los principios y la identidad cooperativa. Dos temas que marcaron las políticas de la ACI de estas décadas y que jugaron un papel esencial en el posterior reconocimiento del principio de interés por la comunidad.

\subsubsection{Nuevos problemas globales}

El primero de estos temas fue la identificación constante por parte del movimiento cooperativo de nuevos y emergentes problemas globales vinculados a la sociedad y al entorno que les rodea. Unos problemas a los que los organismos internacionales y diferentes movimientos sociales ya les habían otorgado relevancia ${ }^{81}$, pero sobre los que las cooperativas todavía no habían tomado partido en la búsqueda de soluciones. Esta situación varía a partir del Congreso de París de 1976, en el cual el movimiento cooperativo comienza una labor de identificación y de propuesta de soluciones apropiadas. En dicho congreso se aprueba la resolución

78 Fauquet, siguiendo a otros teóricos como Walras, Raiffeisen, Schultze-Delitzsch, Luzzatti o Haas, mostró sus dudas ante la consecución del sueño de la cooperación integral de la economía. En este sentido, Fauquet consideró más realista la implantación de un sector cooperativo que complementara a los sectores públicos y privados de la economía. Esto se evidencia en su obra de 1935 al postular que "ateniéndonos a una visión positiva de la realidad, se ve claramente que ni la cooperación de consumo por sí sola, ni todo el conjunto de formas de la cooperación, pueden invadir el campo todo de la economía. Es bastante con que pretendan ocupar un sector, cuya extensión podrá de otro lado variar según la naturaleza de las fuerzas económicas y políticas del medio circulante y las cualidades de los cooperadores mismos" (Fauquet, 1973:21).

Esta tesis del sector cooperativo fue recogida por la propia ACI, de forma que cuando formula el principio de colaboración cooperativa en 1966, alude a la misma en los términos siguientes: "La idea de un sector cooperativo en la economía es con demasiada frecuencia un concepto intelectual, sin la correspondiente realidad material, simplemente a causa de la falta de unidad y cohesión entre las diferentes ramas del Movimiento" (ACI, 1975:84).

79 De entre esas enmiendas, cabría nombrar las presentadas por la Société Générale Coopérative belga, representada precisamente por Lambert, y la Lega Nazionale delle Cooperative de Italia, que propusieron la introducción de un párrafo que incluyera la irrepartibilidad de las reservas y que en caso de disolución de la cooperativa, "sus activos fuesen entregados a otra sociedad o a alguna organización desinteresada que trabaje por el bien común" (ACI, 1966:184), sin embargo, dichas enmiendas resultan denegadas por una amplia mayoría.

80 Únicamente se introdujo la palabra racial entre las discriminaciones indeseables.

81 Por ejemplo, el problema medioambiental fue de especial trascendencia en los años previos. La ONU celebró en 1972 la Conferencia sobre el medio ambiente en Estocolmo que condujo al establecimiento del Programa de las Naciones Unidas para el Medio Ambiente, a la vez que otros muchos movimientos sociales se habían organizado para presionar a los gobiernos tratando de conseguir actuaciones tendentes a su protección. 
de la Asamblea General en la que se fijan los primeros cuatro problemas globales a los que debe enfrentarse el cooperativismo, estos son: el medioambiente; la energía y el consumo excesivo de recursos no renovables; el aumento de población y el agotamiento de los recursos; y la escasez de comida (ACI, 1978).

En el siguiente congreso de la ACI, el celebrado en Moscú en 1980, se presenta el informe de Laidlaw (1981) titulado Las cooperativas en el año 2000, un trabajo encargado para coordinar los pronósticos de los posibles cambios que tendrían lugar durante los siguientes 20 años y determinar cuáles serían las condiciones en las que operarían las cooperativas. En dicho informe, Laidlaw identifica inicialmente 10 problemas a los que se enfrenta el mundo de la época, entre los que se encuentran los problemas sociales, energéticos y de recursos, poblacionales y de alimentos, laborales, medioambientales ${ }^{82}$. Aunque finalmente, el autor indica que la labor de las cooperativas se ha de concentrar en cuatro prioridades: alimentación, empleo, distribución de los bienes de consumo y el contexto de la comunidad, estableciendo además los diferentes papeles que pueden desarrollar las cooperativas en cada una de ellas (Laidlaw, 1981).

En el Congreso de Hamburgo de 1984 se presenta el informe sobre Las cooperativas y los problemas globales de nuestro tiempo de Trunov (1984), en el que, tras realizar una encuesta a varias organizaciones cooperativas, consigue identificar los problemas mundiales que las cooperativas consideran primordiales y establece el orden de prioridad que le otorgan a cada uno de ellos. Trunov concluye que para las cooperativas los principales problemas globales en orden de prioridad son: la paz, la alimentación, el problema energético, la protección medioambiental y el atraso de los países en desarrollo ${ }^{83}$ (Trunov, 1984).

Como puede observarse, a lo largo de estos Congresos hay temáticas que se repiten como son el medioambiente, los alimentos, la energía, los recursos no renovables, el empleo... La creciente preocupación por todos estos temas, junto con la aparición del fenómeno del desarrollo sostenible en $1987^{84}$, ejercieron una influencia fundamental en la posterior manifestación del principio de interés por la comunidad.

\subsubsection{La búsqueda de la identidad cooperativa}

Mientras que el segundo tema que acaparó los congresos de la ACI fue el problema de la continua pérdida de identidad cooperativa. Esto se puso en evidencia en primer lugar en el informe realizado por Laidlaw en el que hablaba de la tendencia actual de los socios y los dirigentes de cooperativas de priorizar el "ir bien con los negocios" en lugar de atender a la teoría e ideología cooperativa (Laidlaw, 1981:28). En dicho informe establece que las cooperativas, en base a su identidad, han de cumplir con un doble propósito, tanto la satisfacción de objetivos sociales como económicos, tratando de encontrar el equilibrio en la satisfacción de ambos. Las cooperativas al anteponer la eficiencia económica a la eficiencia social, traicionan uno de los pilares fundamentales de la filosofía cooperativa como es ese doble propósito. Además de la identificación de esta desviación, Laidlaw también presentó algunas incertidumbres que provocaba la actual declaración de principios. En este sentido, consideraba que se habían elevado las prácticas del momento al nivel de principios y, en su lugar, se debería de haber identificado el principio mismo, es decir, sostenía que en la declaración se había reconocido el aspecto exterior y no la esencia de Rochdale (Laidlaw, 1981).

Ante las inquietudes surgidas a lo largo de la década de los 80 con respecto a la identidad cooperativa, el Comité ejecutivo de la ACI le encarga a Lars Marcus la elaboración de un informe que es presentado en el Congreso de Estocolmo de 1988 titulado Co-operatives and Basic Values. En él, Marcus vuelve a llamar la atención sobre el acercamiento de las cooperativas a objetivos exclusivamente económicos, actitud que es consecuencia de la malinterpretación que están haciendo estas del concepto de eficiencia en los negocios cooperativos $^{85}$ y causando, a su vez, un desgaste de la identidad cooperativa. En relación a la identidad cooperativa, Marcus defiende que la filosofía cooperativa no está formada exclusivamente por principios, en este sentido observa que los principios no indican cómo han de actuar las cooperativas cuando cambian las condiciones del entorno, por lo que establece que los principios y la ACI están construidos sobre unos cimientos que son los valores básicos que los guían ${ }^{86}$. Los valores que Marcus identifica son: participación de los socios, democracia, honestidad y preocupación por los demás, señalando, además, que del estudio de

82 Con respecto a este último, Laidlaw sentencia que: "sea lo que fuere lo que se diga acerca del siglo que está llegando a su fin, debe ser recordado como el período en el cual la humanidad más ha hecho para envenenar y destruir el medio ambiente, más que en todas las épocas previas de la historia" (Laidlaw, 1981:21)

83 Aunque Trunov advierte de que el único en el que hay unanimidad en el orden de prioridad es el problema relativo a la paz, existiendo diferencias a la hora de asignar los siguientes puestos. Además, indica que los representantes de los movimientos cooperativos también mencionaron otros problemas que a su parecer tenían un carácter global, entre los que se encontraban la democratización de la sociedad, los derechos humanos, la situación de las minorías religiosas y de las minorías raciales, problemas de la manera de economizar y racionalizar la energía, las materias primas y otros materiales, la erradicación de enfermedades virulentas, la provisión de agua potable, etc. (Trunov, 1984).

84 El concepto de desarrollo sostenible surgió del informe titulado Our Common Future, más conocido como Informe Brundtland, presentado en la ONU en 1987, en el que se define por primera vez dicho término como aquel que "satisface las necesidades de la presente generación sin comprometer la capacidad de las futuras generaciones para satisfacer las suyas".

85 Marcus manifiesta que es muy difícil ver dónde empiezan o acaban los asuntos económicos, pero "el objetivo final de un miembro de una cooperativa no debería ser sólo el beneficio económico sino algo más: una vida más rica, una vida más segura, una vida más digna, paz y derechos humanos, defensa de la democracia y solidaridad con los no afortunados, dondequiera que se encuentren"(Marcus, 1988:99).

86 Expresa Marcus (1988:96) que son "unos valores básicos de los que se viene hablando durante prácticamente los 28 Congresos anteriores". 
los valores puede surgir la necesidad de reformular los principios cooperativos. Es precisamente el último de los valores identificados por Marcus, la preocupación por los demás, el que presenta una mayor afinidad con los problemas de eficiencia social que sufrían las cooperativas y con el principio de interés por la comunidad. Sobre este valor Marcus dice que "preocuparse de los demás es un valor básico que proviene de nuestra naturaleza como organización de autoayuda y no es algo calculado para atraer clientes. Eso es lo que marca la diferencia entre nosotros y las actividades puramente orientadas al beneficio" (Marcus, 1988:101). El contenido de este informe fue adoptado por unanimidad por la ACI, reconociendo el valor básico de preocupación por los demás entre los valores que guían al movimiento cooperativo. A su vez, se exteriorizó la inquietud existente por el debilitamiento de la identidad cooperativa, por lo que el Congreso encargó a Böök la elaboración de un trabajo sobre cooperación y valores fundamentales que permitiera encontrar las bases, los valores básicos, del sistema cooperativo futuro.

En el Congreso de 1992 de Tokio, Böök presenta su informe titulado Valores cooperativos para un mundo en cambio donde se delimitan los valores que han de guiar el funcionamiento de las cooperativas y la aplicación de sus principios ${ }^{87}$. Tras analizar las ideas, la ética y las prácticas del cooperativismo tradicional ${ }^{88}$, finaliza el estudio con la identificación de cinco valores básicos globales para el futuro de las cooperativas, valores que "son la esencia del sistema cooperativo, de su base organizativa, de sus propósitos y de sus relaciones comunitarias" (Böök, 1992:15) ${ }^{89}$. Así Böök recomienda que, a modo de valores, las cooperativas apliquen: actividades económicas para satisfacer necesidades; democracia participativa; movilización de los recursos humanos; responsabilidad social; y cooperación nacional e internacional.

De esos cinco valores globales cabría destacar, por su relación con los intereses de la comunidad, la responsabilidad social ${ }^{90}$. Böök le otorga una especial relevancia a este valor al decir que desde la perspectiva de justicia económica y social "todos los valores cooperativos básicos están impregnados por la responsabilidad con la comunidad en general" (Böök, 1992:205). Esto se debe a que el objetivo de la creación de las cooperativas, tanto en el pasado como en el presente, es contribuir a la sociedad y mejorar las condiciones de la gente y, para ello, las cooperativas actúan adoptando una organización dual ${ }^{91}$. Por un lado, son organizaciones económicas, y cuando operan como tal en el mercado no pueden actuar con el único objetivo de lograr el máximo beneficio económico para los socios, ni convertirse en explotadoras, sino que han de ser vistas como una opción de mercado en la que la gente puede confiar al ofrecer alternativas buenas, abiertas y honestas, actuando como correctoras del mercado cuando este falle en satisfacer necesidades esenciales. Y por otro lado, también son organizaciones sociales y, como tales, tienen que corregir los fallos sociales. En este sentido, el informe reconoce que existe un problema de identidad en las cooperativas, las cuales tienden cada vez más a asimilarse a sociedades de capital, priorizando la eficiencia económica sobre la ideología cooperativa, es decir, obviando la responsabilidad social. Para Böök las cooperativas, a través de decisiones tomadas democráticamente por los $\operatorname{socios}^{92}$, siempre han realizado políticas sociales que van más allá de satisfacer exclusivamente las necesidades de los socios, ya sea a través de la efectiva multiplicación de la democracia, la participación y la movilización, o promoviendo el valor de la responsabilidad social en las actividades normales de la cooperativa o en el uso de excedentes e ingresos, por lo que debía reconocerse el valor de la responsabilidad social como parte fundamental de la identidad cooperativa. Además, a esta pérdida de identidad han contribuido unos principios cooperativos no actualizados, no universales y que, tal

87 Böök resume los acontecimientos sucedidos en los Congresos precedentes mediante un enunciado extraordinario: “Alguien dijo una vez que el sector cooperativo mundial identificó sus principales áreas para el futuro en Moscú, sus principales perspectivas en Hamburgo y su "alma" en Estocolmo. Ahora en Tokio es tiempo de juntar todo y comenzar a moldearlo en guías instrumentales básicas para el futuro" (Böök, 1992:195).

88 El autor comienza el estudio identificando y clasificando los valores tradicionales cooperativos en tres categorías principales: ideas básicas, éticas básicas y principios básicos. Examinando cada una de estas categorías se observa que, en el interior de todas ellas, el autor sitúa valores tradicionales que han ostentado las cooperativas durante toda su historia y que reflejan su preocupación por los intereses de la sociedad. Así, dentro de las ideas básicas establece que las cooperativas deberían caracterizarse por su responsabilidad ante toda la comunidad; entre las éticas básicas indica que los cooperativistas deben interesarse y preocuparse por otras personas y responsabilizarse por el impacto de su actividad en la sociedad; mientras que en los principios básicos habla del carácter dual de las cooperativas.

89 Para Cracogna (1993:90) este movimiento de identificación de los valores cooperativos "constituyó un positivo catalizador orientado a la búsqueda de la raíz común de la identidad cooperativa".

90 Cuando Böök (1992) analiza en el informe cómo han de implementar las cooperativas cada uno de estos valores, se observa como en algunos de ellos también se encuentran referencias a actitudes que propugnan la inclusión de intereses sociales, ajenos a los socios, entre las prácticas que han de realizar las cooperativas. Así lo vemos cuando hace referencia a la protección del medio ambiente dentro del valor de economía para las necesidades, o la referencia a la participación de los empleados en la toma de decisiones en el valor de democracia participativa.

91 Este carácter dual de la cooperativa tiene su origen en la teoría que fue elaborada por Fauquet, el cual identifica a las cooperativas como entidades duales formadas por 2 elementos constitutivos, la asociación de personas que constituyen la sociedad, que se corresponde con el aspecto humano y social de la cooperación; y la empresa común por medio de la cual alcanza la satisfacción de las necesidades de sus miembros. Este carácter dual de las cooperativas había sido cuestionado en los últimos años por una parte del movimiento cooperativo que defendía la necesidad de priorizar la eficiencia económica sobre la social (Fauquet, 1973).

92 Böok hace hincapié en que las políticas y qué hacer con los recursos de la cooperativa es decisión exclusiva de los socios, sin que se pueda permitir la interferencia de un gobierno, ya que esta actitud dañaría la responsabilidad social y sus caracteres básicos como son la democracia y la autoayuda (Böök, 1992:182). Es reseñable esta postura ya que cuando se apruebe en 1995 el principio de interés por la comunidad, se dejará total libertad a los socios para establecer las medidas y políticas orientadas a la satisfacción del mismo. 
y como expresaba Laidlaw, están demasiado orientados a la práctica y poco hacia los valores esenciales, por lo que aboga claramente por una seria revisión de los valores y principios cooperativos ${ }^{93}$.

Como ha podido verse, la preocupación por la pérdida de identidad cooperativa y el acercamiento hacia objetivos exclusivamente económicos es una constante en todos estos informes. Además, todos ellos reconocen la existencia de una actitud o valor en la tradicional forma de actuar de las cooperativas que muestra un interés especial por la sociedad que la rodea, Marcus lo denomina como el valor de preocupación por los demás, mientras que Böök lo llama el valor de responsabilidad social, término que adopta la ACI como valor en 1992. La conjunción de ambas cuestiones influirá en gran medida en el posterior reconocimiento del principio de interés por la comunidad.

\subsection{El reconocimiento como principio cooperativo autónomo del interés por la comunidad en la Declaración sobre la identidad cooperativa (1995)}

La ACI finaliza el Congreso de 1992 por un lado, mostrándose realmente preocupada por problemas globales como el medioambiente ${ }^{94}$, el problema de alimentos, el aumento de población, etc. tal y como se había escenificado en los congresos celebrados a lo largo de los quince años previos, a la vez que observa como estos problemas han adquirido una elevada relevancia tanto para la sociedad, como para otros organismos internacionales como la ONU, cuyos últimos congresos han llevado como temáticas principales esos mismos problemas $^{95}$. Y por otro lado, encontramos una ACI en la que se ha hecho patente la necesidad de actualizar los principios cooperativos a otros más universales, y que estén en concordancia con los valores adoptados en 1992, entre los que se encuentra la responsabilidad social. Estas circunstancias propiciaron, sin ninguna duda, la aparición en el Congreso de Manchester de 1995 del principio de interés por la comunidad ${ }^{96}$.

A raíz de todos estos hechos, se le encarga a MacPherson la remodelación de los principios cooperativos. Durante la elaboración del borrador del documento final, la cuestión de introducir un principio que recogiera la preocupación por la comunidad de las cooperativas no supuso ninguna controversia, siendo este un punto ampliamente aceptado ${ }^{97}$. Finalmente, el trabajo definitivo fue presentado en el Congreso centenario de la ACI celebrado en Manchester en 1995, donde fue aprobada la Declaración de la ACI sobre la identidad cooperativa ${ }^{98}$. En esta declaración, la ACI le otorga una autonomía propia e independiente a la preocupación de las cooperativas por la comunidad y vuelve a reconocer, aunque con un mayor énfasis, lo que ya hizo en la anterior declaración de principios de 1966, que las cooperativas buscan el beneficio tanto de sus socios como de las comunidades en las que se insertan. Este reconocimiento se materializa en el actual séptimo principio de interés por la comunidad cuyo contenido es: "Las cooperativas trabajan para conseguir el desarrollo sostenible de sus comunidades mediante políticas aprobadas por sus socios"(ACI, 1995:19). A través de este principio, se manifiestan tanto los valores cooperativos de responsabilidad social, proveniente del trabajo de Böök, como el de preocupación o cuidado por los demás, el cual recogía Marcus en su trabajo.

93 Sobre la revisión de los principios, Böök (1992) plantea dos modalidades de reforma. La primera es una reforma modesta consistente en la reformulación de los principios existentes y en la que se centra en aspectos como el interés limitado, el origen del capital, la autonomía y la participación de los trabajadores en la toma de decisiones. En esta modalidad de reforma no incluye un tratamiento especial para la responsabilidad social, solamente el último de los aspectos señalados puede influir al hablar de que en la toma de decisiones se consideren otras posturas diferentes a los de los socios. Mientras que la segunda modalidad consiste en una reforma más ambiciosa, en la que la ACI desarrolle dos categorías de principios, los Principios Cooperativos Básicos y Prácticas Cooperativas Básicas. No habla de cuáles serían los principios a introducir en cada una de esas categorías, pero, a la vista de la importancia otorgada a lo largo del informe a la responsabilidad social de la cooperativa, se puede presumir que dicho carácter tendría una presencia notable entre los Principios Cooperativos Básicos.

94 Además del reconocimiento del problema medioambiental realizado en los informes de los congresos precedentes, cabría señalar que el Congreso de Tokio planteó como tema principal del debate el problema del desarrollo sostenible y el medioambiente, aprobando al finalizar el mismo la Declaración sobre el Medioambiente y el Desarrollo sostenible. Además, en el año 90 por el Día Internacional de la Cooperación, la ACI realiza un manifiesto dedicado al medioambiente. En ese mismo año, en la reunión del Comité Central de la ACI celebrada en Madrid se adoptó una resolución en la que expresaba su preocupación por el estado crítico del medio ambiente y cuestiones del desarrollo.

95 En el marco de la ONU, además del reconocimiento del concepto de desarrollo sostenible realizado en 1987 a través del informe Brundtland, se celebró la Conferencia sobre Medio ambiente y Desarrollo en 1992, denominada la Cumbre de la Tierra, en la que se adoptó la Agenda 21 y la Declaración de Río con la presentación de una serie de principios relacionados con el desarrollo sostenible, la vida saludable y productiva en armonía con la naturaleza, etc. Posteriormente, se celebró la Cumbre Mundial de la ONU sobre Desarrollo Social en 1995, en la que se hizo referencia a la importancia de las cooperativas para lograr algunos objetivos primordiales del desarrollo sostenible como son la erradicación de la pobreza, la meta del pleno empleo y el fomento de la integración social, debido al gran número de personas que involucran a su alrededor.

96 La ACI reconoce el vínculo existente entre la Conferencia de la ONU denominada Cumbre de la Tierra de 1992 y el reconocimiento del principio de interés por la comunidad en 1995 (ACI, 2016). Asimismo, Paz Canalejo (1995) reconoce que este el principio proviene del Congreso de Tokio donde se recogió la prioridad de los problemas medioambientales y, como trasfondo de dicha prioridad, la idea de responsabilidad social.

97 MacPherson reconoce que en los intercambios de opinión entre expertos cooperativistas llevada a cabo durante la elaboración del borrador de los principios, "la "preocupación por la comunidad" era un tema común, un principio ampliamente aceptado que se creía que el movimiento internacional debía reconocer y aplicar en su labor. Al final del proceso de consulta no había duda de que las asociaciones comunitarias de cooperativas y el movimiento cooperativo eran de vital importancia en la mente de los cooperadores comprometidos" (MacPherson, 2012:5)

98 Lo interesante de este documento no es únicamente la modificación de los principios, sino que esta se lleva a cabo dentro de una Declaración de identidad en la que, a la vez, se incluye la primera definición en la historia de la ACI de qué es una cooperativa, junto con los valores cooperativos. De forma que todo lo que contiene este documento ayuda a comprender la filosofía cooperativa en su conjunto, a diferencia de lo que ocurría con las anteriores aisladas declaraciones de principios. En este mismo sentido opina MacPherson (1995) 
El Comité también destacó la trascendencia de este principio debido a la importante influencia que ejercen los lazos comunitarios sobre todos los valores cooperativos ${ }^{99}$ (MacPherson, 2012).

En la definición del principio se encuentran algunas palabras y expresiones cuya elección fue explicada años después por MacPherson (2012) ${ }^{100}$. Así, según su autor, la palabra sostenible tiene su base en las iniciativas de cooperativistas de zonas rurales y urbanas en declive, donde se desarrollaron cooperativas para fortalecer las comunidades, aunque también reconoce que influyó la unanimidad con respecto al significado de la idea de sostenibilidad y cómo podía lograrse, como consecuencia de la difusión realizada del informe Brundtland por parte de la ONU. Por otro lado, en la última expresión mediante políticas aprobadas por sus socios, se aprecia una continuidad con las ideas expuestas por Böök cuando se refería al valor cooperativo de responsabilidad social. Sobre esta expresión MacPherson indica que se incluyó para asegurar la autonomía e independencia de las cooperativas, evitando que grupos externos trataran de manipularla para sus propios intereses. A la vez, se declaraba el deber que tienen los consejos rectores de conseguir que se aprueben iniciativas comunitarias y de alentar al debate entre los socios sobre cómo debía su cooperativa relacionarse con sus comunidades, buscando un equilibrio entre el interés propio y el interés de la comunidad.

La Declaración va acompañada de un informe en el que se define con una mayor concreción el contenido y significado de cada uno de los principios. En dicho documento se aclara que las cooperativas tienen una responsabilidad para asegurar el desarrollo de sus comunidades en los ámbitos económico, social y cultural y una responsabilidad para la protección medioambiental, siendo los socios los únicos competentes para decidir en qué profundidad y de qué forma debe hacer la cooperativa las aportaciones a la comunidad (ACI, 1995) ${ }^{101}$. Pero uno de los aspectos más sorprendentes de este informe es que apenas le dedica un párrafo a la ampliación y clarificación del principio de interés por la comunidad, mientras que al resto de principios le dedica como mínimo una página completa.

\subsection{La interpretación vigente del principio de interés por la comunidad tras las Notas de orientación para los principios cooperativos (2016)}

En los últimos años se ha producido en el interior de la ACI un intento de modificar este séptimo principio. Esta corriente se inició en la Asamblea general extraordinaria de la ACI, celebrada en Cancún en 2011. Tras algunos acontecimientos relevantes que provocaron la aceleración del cambio climático y aumentaron el daño medioambiental ${ }^{102}$, la Asamblea propuso una enmienda al séptimo principio que reconociera la importancia de la sostenibilidad ambiental y la necesidad de una gestión responsable de los recursos naturales, la riqueza, la conservación de la biodiversidad y la mitigación de los cambios climáticos. Por ello propuso, en primer lugar, modificar el nombre del $7^{\circ}$ principio, pasándose a llamar "Preocupación por la comunidad y la sostenibilidad ambiental"; y en segundo lugar, modificar su contenido por la siguiente redacción: "Las cooperativas trabajan para el desarrollo sostenible de sus comunidades, incluida la protección del medio ambiente, mediante políticas aprobadas por sus miembros, promoviendo la gestión responsable de los recursos naturales para garantizar el equilibrio ecológico" (ACI, 2011:13).

La resolución sobre la propuesta de enmienda fue expuesta para su aprobación ante la Asamblea General extraordinaria de la ACI celebrada en Manchester en 2012, sin embargo no se acordó aprobarla, sino que se acordó transmitir de nuevo dicha petición a la Junta de la ACI, resolviendo esta posteriormente crear el Comité de Principios y encargarle la tarea de elaborar unas notas orientativas sobre el tercer, quinto y séptimo principio (ACI, 2012). Dicho comité debía establecer una orientación para los principios cooperativos tal y como estaban redactados en el momento, así como delimitar el núcleo irreductible de cada principio, y por lo tanto, de la identidad cooperativa.

En el año 2013 se presentó el borrador de dicho trabajo, el cual incluye unas notas de orientación sobre el séptimo principio ${ }^{103}$. Este trabajo hace alusión a las mismas dimensiones que había de implicar la sostenibilidad y que habían sido señaladas en el informe que acompañaba a la Declaración de 1995, pero elimina de ellas la dimensión cultural. De forma que las dimensiones de la sostenibilidad resultan ser la

99 En este sentido, MacPherson (2012) hace una recopilación de la influencia de los lazos comunitarios sobre cada uno de los valores cooperativos aprobados en esa misma Declaración de identidad.

100 Unas explicaciones parecidas a las realizadas por MacPherson sobre cómo se han de interpretar estas palabras y frases se recogen también en las Notas de orientación para los principios cooperativos (ACI, 2016) que fueron publicadas en 2016 y de las que hablaremos más adelante.

101 En este mismo Congreso se presenta el informe titulado Las cooperativas y el desarrollo humano sostenible que armoniza a la perfección con este principio, así lo reconoce el propio MacPherson (1995). Dicho informe, fue elaborado a raíz de la resolución de la Asamblea General de la ONU de 1994 que reconoce la contribución de las cooperativas al desarrollo humano sostenible, e incluye algunas referencias a la importancia de tomar en consideración las cuestiones ambientales y la propuesta de la Agenda Cooperativa 21, la cual contiene propuestas de actuaciones en materia ambiental que pueden realizar cada uno de los sectores cooperativos (ACI, 1995b).

102 Se había producido la renuncia de algunos países al cumplimiento de los acuerdos de la Cumbre de la Tierra, el Protocolo de Kyoto, los Objetivos de Desarrollo del Milenio y las recomendaciones formuladas por diversos foros mundiales.

103 Dante Cracogna fue el encargado de elaborar el borrador inicial sobre las notas de orientación del séptimo principio. 
económica, la social y la medioambiental. Además, siguiendo la línea marcada desde el 2011, se subraya la preponderancia de la sostenibilidad medioambiental sobre las demás dimensiones ${ }^{104}$ (ACI, 2013).

Finalmente, en el año 2016 el Comité de Principios presentó el documento final titulado Notas de orientación para los principios cooperativos (ACI, 2016), el cual incluía unas guías de interpretación de los siete principios cooperativos que dejaban constancia de cómo han de aplicarse los principios en términos adaptados al siglo XXI. De manera que en este documento se encuentra la indicación más reciente de cómo ha de interpretarse en la actualidad el principio de interés por la comunidad. En este sentido, el informe señala que el desarrollo sostenible de sus comunidades al que se refiere dicho principio, engloba por igual a las tres dimensiones: el equilibrio ecológico, la justicia social y la seguridad económica y añade que "al ser interdependientes y de regeneración mutua deberán perseguirse de manera concomitante" (ACI, 2016:94). Así pues, a diferencia del borrador previo, ninguna de las tres dimensiones del desarrollo sostenible se sobrepone a las demás, sino que todas deben tener la misma importancia y complementarse mutuamente.

Además, para facilitar aún más la interpretación de estos términos, las guías de orientación contienen una explicación de los ámbitos que deben abarcar cada una de esas tres dimensiones, así como una serie de actitudes observadas en algunas cooperativas que sirven de ejemplo para entender a qué se refiere o qué conductas incluyen cada una de ellas. Así, la ACI, con respecto al desarrollo económico sostenible, establece que no consiste en intentar obtener el máximo índice de rentabilidad en los negocios, sino en tratar de satisfacer las necesidades de sus socios a la vez que se aplican valores éticos a las operaciones comerciales y se realizan actuaciones encaminadas a reducir la pobreza, las desigualdades de renta, a conseguir el pleno empleo y la integración social. Por otro lado, en cuanto a la sostenibilidad ambiental, el informe indica que las cooperativas, además de reducir el impacto medioambiental en sus actividades, deben contribuir activamente a sensibilizar a otros colectivos, ya que la magnitud del problema exige abordarlo de manera conjunta y coordinada desde distintos sectores.

Sin embargo, al que más atención dedica es al apartado destinado al desarrollo social sostenible. En él, la ACI engloba una amalgama muy variada de actuaciones, llegando a configurar una especie de cajón de sastre de conductas que en general favorecen a la comunidad, pero que, por su contenido, no es posible encuadrarlas dentro de las actuaciones de desarrollo económico y medioambiental. Incluso llega a encuadrar actuaciones que presentan una relación más estrecha con otros principios como el de intercooperación o el de educación, pero que, al generar una influencia positiva en la comunidad, decide recogerlas también como actuaciones pertenecientes a este séptimo principio.

Así, la ACI entiende que para favorecer el desarrollo social sostenible, las cooperativas deberán dar respuesta a necesidades y aspiraciones intangibles de la comunidad como "la cultura y las artes, la espiritualidad y los derechos religiosos, la educación, la historia y el patrimonio, los festivales comunitarios y culturales, así como las artes visuales". A su vez, también señala el historial que tienen las cooperativas en satisfacer otras necesidades sociales "mediante la prestación de servicios sanitarios, de vivienda, educativos, sociales, de integración en el mercado laboral de personas socialmente desfavorecidas, y de ayudar al desarrollo de la comunidad"105 (ACI, 2016:94). Sin embargo, con respecto a esta última apreciación, el simple ejercicio de este tipo de actividades económicas, aunque pretendan satisfacer necesidades sociales, no debería ser considerado como un especial interés por la comunidad, sino que será necesario que a través de esta actividad se satisfaga una necesidad social no atendida por el mercado ${ }^{106}$, o bien, que la actividad se realice de forma acorde a la interpretación de la dimensión económica del desarrollo sostenible ${ }^{107}$.

Dentro del desarrollo social, la ACI también encuadra la asistencia a cooperativas en países en desarrollo, actuación que podría estar más relacionada con el sexto principio de intercooperación, pero que, sin duda, influye de manera positiva en el desarrollo social de la comunidad desfavorecida. También sitúa dentro de este ámbito otros aspectos más generales y en los que la cooperación lleva años comprometida como es el trabajo por la paz y la justicia social, así como la promoción y el apoyo a los jóvenes, promoviendo, por ejemplo, el acceso de estos a los consejos rectores, fomentando actividades de jóvenes y organizaciones de juventud cooperativa, e impulsando la educación cooperativa en colegios, institutos y universidades ${ }^{108}$.

104 Aunque en un primer momento indica que las tres dimensiones se han de complementar entre sí, más adelante señala la primacía de la sostenibilidad medioambiental debido a los fenómenos producidos en los últimos años de degradación del medio ambiente y de aumento de la población mundial. Igualmente, concluye el borrador advirtiendo de nuevo de la gravedad del problema medioambiental y la importancia de la aplicación eficaz de este séptimo principio para tratar de solucionarlo (ACI, 2013).

105 La ACI destaca en este sentido como referente a las cooperativas sociales italianas, cuyo objeto social es una misión de interés general, a través de la producción de bienes o servicios de interés general y donde los beneficiarios son sus miembros y la comunidad en general (ACI, 2016).

106 Esto es algo que identificó Marcus (1988) cuando analizaba el valor de preocupación por los demás. Señalaba que en las cooperativas siempre se hallaban algunas cualidades sociales que surgían ante la negligencia de la sociedad a la hora de satisfacer necesidades sociales.

107 En este sentido, pensemos en un hospital privado que establece unas tarifas elevadas ¿podría decirse que se muestra preocupado por la comunidad por el hecho de prestar un servicio sanitario? El interés por la comunidad no se refleja en el tipo de actividad económica realizada, sino en la forma en la que se realiza, ya sea contratando a personas en riesgo de exclusión, con tarifas asumibles por la mayoría de la población...

108 Al igual que ocurría anteriormente, estas últimas actuaciones parecen más relacionadas con otro principio, el de educación, aunque no cabe duda del beneficio para la comunidad que pueden generar este tipo de intervenciones. 
Por último, también se incluye enmarcado dentro del desarrollo social, como ámbito de especial interés a adoptar por las cooperativas, la preocupación y la mejora de las condiciones laborales y el bienestar de sus empleados y sus familias. En este sentido, el texto hace referencia a la adopción por parte de la ACI de la Recomendación 193 de la Organización Internacional del Trabajo de 2002 sobre la Promoción de las Cooperativas y a la obligación que tienen las cooperativas de respetar los convenios, recomendaciones y normas laborales de la OIT, las cuales deben ser "el punto de partida para las políticas de empleo de una cooperativa" (ACI, 2016:96). Suponemos que al hablar de punto de partida se refiere a que las cooperativas no deben conformarse con lo estipulado en esas normativas, sino que deben aspirar a mejorar las condiciones laborales de los trabajadores más allá de lo recogido en la legislación. De lo contrario, cumplir con lo establecido en un convenio laboral no puede decirse que sea mostrar un especial interés por la comunidad, sino cumplir con la legalidad, actitud que deben mostrar todos los empleadores.

\section{Consideraciones finales}

Tal y como se ha podido comprobar, el interés por la comunidad se encuentra presente en el movimiento cooperativo desde su inicio. Incluso mucho antes de que surgiera la cooperativa de Rochdale, los socialistas utópicos que sentaron las bases de lo que posteriormente terminaría convirtiéndose en el movimiento cooperativo moderno, ya mostraban una gran vocación social. Tras ellos, las primeras experiencias cooperativas continuaron orientando parte de sus actuaciones a los intereses de la comunidad, trabajando por el bien general de la comunidad.

En cambio, estas prácticas no recibieron inicialmente la atención suficiente por parte de la ACI, lo cual se evidencia en los primeros principios cooperativos de 1937, entre los cuales no hay ninguna referencia a la defensa de unos intereses distintos a los de los socios. Esta situación varía ligeramente en la segunda formulación de los principios cooperativos de 1966, en los que se incluye en la redacción del principio de cooperación entre cooperativas, una referencia a la obligación que tienen las cooperativas de servir a los intereses de sus miembros y de las comunidades de las que forma parte.

Sin embargo, hay que esperar a que los problemas medioambientales y el concepto de desarrollo sostenible estén totalmente asentados en la mayoría de los debates mundiales, y una vez que otros movimientos, como el de la Responsabilidad Social Corporativa, que pretenden ocuparse de que las empresas cumplan con esas demandas, han alcanzado una gran trascendencia, para que la ACI le otorgue al interés por la comunidad la categoría de principio cooperativo. Estas circunstancias, unidas a las insuficientes aclaraciones y escasa atención que le presta a la preocupación por la comunidad el informe que acompaña a la Declaración de 1995, generan la impresión de que la ACI decide poner en relevancia la defensa que realizan las cooperativas de los intereses de la comunidad, con la mera intención de situar al movimiento cooperativo como uno de los principales defensores de dichas demandas.

A la vista del exiguo desarrollo de dicho principio, la ACI en 2016 publicó las Notas de orientación para los principios cooperativos, en las que precisó que el desarrollo sostenible de las comunidades engloba tres ámbitos diferenciados: el ecológico, el social y el económico. En esta ocasión trata de aclarar el contenido del principio identificando actuaciones cooperativas que encajan dentro de esos ámbitos. Pero el problema surge cuando para desarrollar la dimensión social genera un totum revolutum de prácticas cuyo único criterio para clasificarlas como tal es que generen un beneficio a la comunidad. Esta decisión provoca que, en la actualidad, prácticamente cualquier conducta que procure un beneficio en el medioambiente o a otro colectivo diferente al de los socios, y a pesar de que presenten una relación más estrecha con otro principio como el de educación o intercooperación, va a ser catalogada como perteneciente al séptimo principio. De forma que, gracias a esta amplitud de conductas, las cooperativas van a encontrar muchas facilidades a la hora de justificar el cumplimiento de este principio y de demostrar su aspecto más social.

Con el presente artículo no finaliza mi investigación en este ámbito, sino que se trata de la primera parte de una investigación de más largo recorrido. En un futuro próximo, se espera poder ofrecer al lector una segunda publicación en esta misma revista, que dará continuidad a este trabajo y que versará sobre la recepción jurídica del principio cooperativo de interés por la comunidad tanto en el Derecho positivo español, estatal y autonómico, como en Derecho comparado. En base en las diferentes formas de afrontar la inclusión de este principio en la legislación y al grado de compromiso con los intereses de terceros que impongan, se efectuará una clasificación de las legislaciones cooperativas en más o menos sociales, a la vez que permitirá conocer si es posible identificar diferentes modelos legislativos cooperativos en función del tratamiento de este principio. 


\section{Referencias bibliográficas}

ACI (1895) Report of the First International Cooperative Congress held in the Hall of the Society of Arts, on 19th, 20th, 22nd and 23rd August 1895. Londres: P.S. King \& Son, S.A.

ACI (1897) Statuts de l'Alliance. En: Deuxième Congrès: tenu au Musée social, 5, rue Las-Cases, à Paris : du 28 au 31 Octobre 1896 :compte rendu officiel. Paris: Imprimerie Nouvelle (Association Ouvrière), pp. 152-155.

ACI (1921) Report of the proceedings of the Tenth Congress of the International Cooperative Alliance held at Basle 22nd to 25th August, 1921. Londres: International Cooperative Alliance, S.A.

ACI (1930) Report of the proceedings of the Thirteenth International Cooperative Congress at Vienna, 25th to 28th August, 1930. Londres: International Cooperative Alliance.

ACI (1934) Report of the special committe on the present application of the Rochdale principles. En: Report of the proceedings of the Fourteenth Congress of the International Cooperative Alliance at London, 4th to 7th September, 1934. Londres: International Cooperative Alliance, pp.131-158.

ACI (1938) Rapport sur l'application presente des principes Rochdalienes. En: Compte Rendu du Quinzième Congrès de l'Alliance Coopérative Internationale á Paris, du 6 au 9 Septembre 1937. Londres: Alliance Coopérative Internationale, pp. 159-192.

ACI (1963) Resolution on the Report of the Central Committee. En: Report of the Twenty-Second Congress at Bournemouth 14th to 17th October, 1963. Londres: International Cooperative Alliance, S.A., pp.101-102.

ACI (1966) Report of the Twenty-Third Congress at Vienna: 5th to 8th September, 1966. Londres: International Cooperative Alliance, S.A.

ACI (1975) Nuevos enfoques de los principios cooperativos en el mundo: informe de la Comisión sobre los "Principios Cooperativos" presentado en el Congreso de la Alianza Cooperativa Internacional, celebrado en Viena (Austria) en setiembre de 1966. Rosario: Idelcoop.

ACI (1978) Report of the Twenty-sixth Congress : Unesco House, Paris (France), 28th September to 1st October 1976. Londres: International Cooperative Alliance.

ACI (1995) Declaración de la ACI sobre la identidad cooperativa =The International Co-operative Alliance statement on the co-operative identity. Vitoria-Gasteiz: Consejo Superior de Cooperativas de Euskadi.

ACI (1995,b) Section III - Co-operatives and Sustainable Human Development - (green section). En: XXXI ICA Congress: Manchester 1995: agenda \& reports. Ginebra: International Cooperative Alliance.

ACI (2011) Meeting Documentation - part 2, Asamblea general extraordinaria de la ACI, Cancún, 2011. Disponible en https://www.ica.coop/sites/default/files/event-attachments/ga2011-official-meeting-documents-ii-843944155.pdf.

ACI (2012) Asamblea general extraordinaria de la ACI: Plan de acción para una década de cooperativas, 31 de octubre de 2012. Disponible en https://www.ica.coop/sites/default/files/GA_2012_Official_Meeting_Docs_ES.pdf.

ACI (2013) Borrador de las Guías Orientativas - ayuda para la interpretación de los Principios cooperativos. Disponible en https://www.ica.coop/sites/default/files/basic-page-attachments/guidance-notes-2013-12-09-sp672707590.pdf.

ACI (2016) Notas de orientación para los principios cooperativos. Disponible en https://www.ica.coop/sites/default/files/publication-files/guidance-notes-es-2107251738.pdf.

Benevides Pinho, D. (1987) Evolución del pensamiento cooperativista. Buenos Aires: Intercoop.

Böök, S.A. (1992) Valores cooperativos para un mundo en cambio, Informe para el Congreso de la ACI, Tokio, octubre de 1992. San José, Costa Rica: Alianza Cooperativa Internacional, Oficina Regional.

Cracogna, D. (1993) La identidad cooperativa en un mundo cambiante. Anuario de Estudios cooperativos, pp.87-96.

Divar Garteiz-Aurrecoa, J. (2011) El cooperativismo de consumo orígenes y actualidad. Boletín de la Asociación Internacional de Derecho Cooperativo, No 45, pp. 217-224. DOI: 10.18543/baidc-45-2011pp217-224.

Divar Garteiz-Aurrecoa, J. (2012) Filosofía de la cooperación económica. Boletín de la Asociación Internacional de Derecho Cooperativo, No 46, pp.147-160. DOI: 10.18543/baidc-46-2012pp147-160.

Fauquet, G. (1973) El sector cooperativo. Buenos Aires: Intercoop Editora.

Gaminde Egía, E. (2015) Los orígenes del cooperativismo. En: Aprendizaje cooperativo sin fronteras. Madrid: Dykinson, pp.51-69.

Holyoake, G.J. (1973) Historia de los pioneros de Rochdale. Zaragoza: Aecoop-Aragón. Escuela Sindical de Gerentes Cooperativos.

Johansson, A. (1927) Problems of Modern Co-operation. En: Report of the proceedings of the Twelfth International Cooperative Congress at Stockholm: 15th to 18th August, 1927. Londres: International Cooperative Alliance, S.A., pp. 173-203.

Kaplan de Drimer, A. \& Drimer, B.(1975) Las Cooperativas: fundamentos, historia, doctrina. Buenos Aires: InterCoop.

Laidlaw, A.F. (1981) Las cooperativas en el año 2000. Revista de Idelcoop, Volumen 8, No 28-29. Disponible en https://www.idelcoop.org.ar/sites/www.idelcoop.org.ar/files/revista/articulos/pdf/81012903.pdf.

Lambert, P. (1961) La doctrina cooperativa. Buenos Aires: InterCoop.

Lambert, P. (1984) Los Principios Cooperativos de 1966. En: Eguía Villaseñor, F. Los principios del cooperativismo de Rochdale a nuestros días. Compilación y notas. San Luis Potosí: Confederación Mexicana de Cajas Populares.

MacPherson, I. (1995) Los principios cooperativos. Revista de la Cooperación Internacional: órgano oficial de la Alianza Cooperativa Internacional, № 28 (3), pp.14-28. 
Macpherson, I. (2012) Cooperative's concern for the community, from members towards local communities' interest. Euricse Working Paper $\mathrm{N}^{\mathrm{o}}$ 46/13. Disponible en https://www.euricse.eu/wpcontent/uploads/2015/03/1358347493 n2284.pdf.

Marcus, L. (1988): Cooperatives and basic values. En: XXIX Congress, Stockholm, July 1988, agenda \& reports, 1988, pp. 95-108.

Martínez Charterina, A. (2016) La cooperativa y su identidad. Madrid: Dykinson.

Mateo Blanco, A. \& Palacio Gómez, A. (1979) Cooperativismo. Zaragoza: Talleres Gráficos La Editorial.

Mateo Blanco, A. (1985) Historia de la reforma de los principios cooperativos. REVESCO. Revista de Estudios Cooperativos, N53, pp. 37-68.

Merino Hernández, S. (2005) Los orígenes del cooperativismo moderno y el socialismo premarxista. Revista vasca de economía social = Gizarte ekonomiaren euskal aldizkaria, $\mathrm{N}^{\circ} 1$, pp. 169-188.

Miranda, J.E. de (2014) De la propedéutica de los principios cooperativos a la intercooperación como pilastra del cooperativismo. Boletín de la Asociación Internacional de Derecho Cooperativo, $\mathrm{N}^{\mathrm{o}} 48$ (diciembre), pp. 149-63. DOI: $10.18543 /$ baidc-48-2014pp149-163.

Mladenatz, G. (1969) Historia de las doctrinas cooperativas. Buenos Aires: InterCoop.

Monzón Campos, J.L. (1989) Las cooperativas de trabajo asociado en la literatura economica y en los hechos. Madrid: Ministerio de Trabajo y Seguridad Social.

Paz Canalejo, N. (1995) Principios cooperativos y prácticas societarias de la cooperación. REVESCO. Revista de Estudios Cooperativos, № 61, pp. 15-34.

Thomas, A. (1924) The Relation between the Different Forms of Co-operation. En: Agenda of the Eleventh International Cooperative Congress at Ghent: 1st to 4th September, 1924. Londres: International Cooperative Alliance, pp. 97-117.

Trunov, M.P. (1984) Las cooperativas y los problemas globales de nuestro tiempo. En: XXVIII Congreso de la Alianza Cooperativa Internacional, Hamburgo, Octubre de 1984. Rosario: Idelcoop, pp. 42-117.

Vargas Vasserot, C. (2015) Las cooperativas de crédito y su posición dentro del modelo cooperativo. Integración frente a diferenciación en el marco de la refoma del sistema financiero. REVESCO, Revista de Estudios Cooperativos, $\mathrm{N}^{\circ}$ 61, pp.50-76. DOI: 10.5209/rev_REVE.2015.v117.48145. 